\title{
Integrative Medicine (Herbal Medicine Combined with Drug Therapy) for Behcet's Disease: A Systematic Review and Meta-Analysis of Randomized Controlled Trials
}

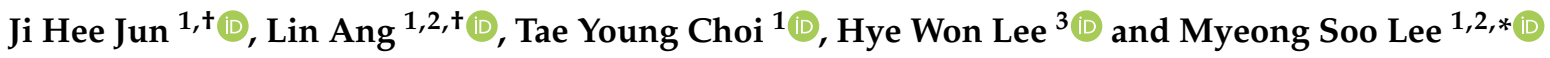 \\ 1 Clinical Medicine Division, Korea Institute of Oriental Medicine, 1672 Yuseongdae-ro, Yuseong-gu, \\ Daejeon 34054, Korea; zhixi04@kiom.re.kr (J.H.J.); anglin2808@kiom.re.kr (L.A.); \\ superoung@kiom.re.kr (T.Y.C.) \\ 2 Korean Convergence Medicine, University of Science and Technology, Daejeon 34113, Korea \\ 3 Herbal Medicine Research Division, Korea Institute of Oriental Medicine, Daejeon 34054, Korea; \\ hwlee@kiom.re.kr \\ * Correspondence: drmslee@gmail.com or mslee@kiom.re.kr; Tel.: +82-42-868-9266; Fax: +82-42-863-9299 \\ + These authors contributed equally to this work.
}

\section{check for} updates

Citation: Jun, J.H.; Ang, L.; Choi, T.Y.; Lee, H.W.; Lee, M.S. Integrative Medicine (Herbal Medicine Combined with Drug Therapy) for Behcet's Disease: A Systematic Review and Meta-Analysis of Randomized Controlled Trials. Pharmaceutics 2021, 13, 476. https://doi.org/10.3390/ pharmaceutics13040476

Academic Editor: Hassan Bousbaa

Received: 11 March 2021

Accepted: 30 March 2021

Published: 1 April 2021

Publisher's Note: MDPI stays neutra with regard to jurisdictional claims in published maps and institutional affiliations.

Copyright: (c) 2021 by the authors Licensee MDPI, Basel, Switzerland. This article is an open access article distributed under the terms and conditions of the Creative Commons Attribution (CC BY) license (https:// creativecommons.org/licenses/by/ $4.0 /)$.

\begin{abstract}
This review aimed to investigate the efficacy of integrative medicine (herbal medicine combined with drug therapy) in the treatment of Behcet's disease (BD). Eleven databases were searched from their inception to 7 December 2020, for randomized control trials (RCTs) that reported the effects of integrative medicine in treating BD. The risk of bias was assessed using seven domain criteria from the Cochrane Collaboration tool. Grading of Recommendations Assessment, Development, and Evaluation (GRADE) was used to assess the quality of evidence. The direction of the effect is also shown in the form of an albatross plot. Sixteen trials met the inclusion criteria and were analyzed. The overall risk of bias was determined to be uncertain. The meta-analysis showed a superior response rate with herbal medicine plus drug therapy (relative risk (RR) 1.19, 95\% confidence interval (CI) 1.13 to $1.25, n=1034, p<0.00001, \mathrm{I}^{2}=0 \%$, low certainty of evidence (CoE)) compared to drug therapy. Integrative medicine also lowered the recurrence rate after 2 months of follow-up (RR $0.27,95 \% \mathrm{CI}$ 0.09 to $0.76, n=120, p=0.01, \mathrm{I}^{2}=0 \%$, low $\mathrm{CoE}$ ). The erythrocyte sedimentation rate (ESR), C-reactive protein (CRP) level, and skin lesions were also significantly improved using integrative medicine, but equivalent effects were seen for oral ulcers, genital ulcers, and eye inflammation. Minor adverse events were reported in both groups. Our findings suggest that herbal medicine combined with drug therapy is more effective for the treatment of BD than drug therapy alone. Although the type of drug therapy used varied across the studies, integrative medicine was shown to improve the total response rate, skin lesions, the ESR, and the CRP level. However, the overall risk of bias of the studies was concerning, and the CoE was low. Information on adverse events (AEs) was also insufficient. In addition, the number of studies included for data synthesis for most outcomes was small. Future studies with rigorous RCTs may help establish the efficacy of integrative medicine in the treatment of BD.
\end{abstract}

Keywords: integrative medicine; complementary and alternative medicine; herbal medicine; Behcet's disease; systematic review

\section{Introduction}

Behcet's disease (BD) is a multisystemic inflammatory disorder characterized by a range of manifestations, such as recurrent oral ulcers, genital ulcers, arthritis, vasculitis, and skin lesions. The age of onset of BD is usually $30-40$ years [1,2]. The prevalence of $\mathrm{BD}$ is higher in the Middle East and Asia than in North America and northern Europe. In particular, its prevalence is highest in Turkey (80-370 cases per 100,000 persons) [3-6]. BD is commonly treated with steroids and immunomodulatory drugs such as corticosteroids, 
colchicine, and thalidomide, which often present side effects such as peripheral neuropathy, loss of appetite, nausea, diarrhea, and intestinal bleeding or perforation [7-9].

Integrative medicine refers to the combination of conventional drug therapies with complementary or alternative medicine (CAM) therapies such as acupuncture and herbal medicine [10]. Integrative medicine combines the benefits of both Western medicine and CAM [11]. It has, therefore, become increasingly prevalent and popular, not only in China but also worldwide [12]. Clinical trials have revealed that a combination of herbal medicine and drug therapy is more effective than herbal medicine alone and significantly reduces the symptoms and recurrence rate of $\mathrm{BD}$. This improvement is accompanied by the expression of the cytokine LI-4 $[13,14]$. A recent publication also reported that herbal medicine was favorable for treating $\mathrm{BD}$, showing the potential of herbal medicine in complementing conventional medication [15].

Two systematic reviews (SRs) have been published on the use of integrative medicine for the treatment of BD $[16,17]$. These SRs included seven randomized controlled trials (RCTs) that compared the effects of integrative medicine with drug therapy; however, the included studies did not have publication dates beyond 2014. Thus, this study aimed to update the evidence on the efficacy of integrative medicine in the treatment of BD.

\section{Methods}

\subsection{Study Registration and Protocol Information}

This review has been registered on PROSPERO CRD4201808496.

\subsection{Data Sources}

Eleven electronic databases, namely, PubMed, Embase, Cochrane Controlled Register of Trials (CENTRAL), China National Knowledge Infrastructure (CNKI), Wanfang Data, VIP Information, OASIS, DBpia, Research Information Service System (RISS), Korean Studies Information Service System (KISS), and KoreaMed, were searched from inception to 7 December 2020. Our search included studies in English, Chinese, and Korean languages. The search strategy included Medical Subject Headings (MeSH) and keywords that reflected the terms related to Behcet's syndrome, BD, Behçet's, integrative medicine, and integrated medicine. Detailed search terms are shown in Supplementary Materials.

\subsection{Study Selection}

\subsubsection{Types of Studies}

Eligible studies included RCTs or quasi-RCTs that compared integrative medicine with drug therapy. Postgraduate theses or dissertations were also eligible. Publications in the form of abstracts, conference proceedings, review articles, or other types of clinical studies, such as nonrandomized controlled studies, case-control studies, case reports and series, and animal studies, were not eligible.

\subsubsection{Types of Participants}

Eligible participants were patients of both sexes and all ages clinically diagnosed with $\mathrm{BD}$. The studies had to meet the following diagnostic criteria for inclusion:

- International Study Group (ISG) criteria [18];

- International Criteria for BD (ICBD) criteria [18];

- Criteria of diagnosis and therapeutic effect of diseases and syndromes in traditional Chinese medicine [19];

- Guidelines for the diagnosis and treatment of BD by the Chinese Rheumatology Association (CRA) [20]; and

- Guiding principle of clinical research on new drugs of traditional Chinese medicine [21].

\subsubsection{Types of Interventions and Comparison}

Studies where patients received herbal medicine combined with drug therapy as an intervention were eligible. Concerning herbal medicine, we included only those studies 
that used herbal decoctions. There were no limitations on the number, administration methods, dosage, or duration of treatment. Studies that integrated other types of CAM therapies, such as moxibustion, acupuncture, massage, and cupping with drug therapy, were excluded.

Studies that used drug therapy alone, no treatment, or placebo as comparators were eligible. Studies in which the comparators included other types of herbal medicine were excluded.

2.3.4. Types of Outcome Measurements Primary Outcomes

- $\quad$ Total relative risk (RR): (recovery + marked improvement + improvement)/total number of cases $\times 100 \%$.

- $\quad$ Recurrence rate.

Secondary Outcomes

- Laboratory changes in C-reactive protein (CRP) levels and the erythrocyte sedimentation rate (ESR);

- $\quad$ Symptom score (oral ulcers, genital ulcers, eye inflammation, skin lesions, and arthralgia); and

- $\quad$ Adverse events (AEs).

\subsection{Data Extraction and Risk of Bias Assessment}

\subsubsection{Data Extraction}

We searched and screened eleven databases by checking the title and abstract. Furthermore, we independently extracted detailed data from the studies pertaining to the seven following domains: (1) first author, year of publication; (2) diagnosis, sample size, duration of treatment; (3) intervention group; (4) control group; (5) main outcomes; (6) results; and (7) AEs. In addition, we obtained the details on the prescription of herbal treatment.

\subsubsection{Risk of Bias}

As an assessment of the quality of each study, two authors (J.H.J and T.Y.C) used Cochrane Collaboration's tool to evaluate the risk of bias [22]. We evaluated seven domains, namely, random sequence generation, allocation concealment, blinding of participants and personnel, blinding of the outcome assessment, incomplete outcome of the data, selective reporting, and other biases, to assess the risk of bias. We categorized the risk of bias as low $(\mathrm{L})$, high $(\mathrm{H})$, or uncertain $(\mathrm{U})$. Disagreements were resolved by another author (MSL).

\subsubsection{Data Analysis}

Data analyses were performed using Review Manager (Version 5.3.5) software provided by the Cochrane Collaboration. We quantified the effects of treatment as the RR with the $95 \%$ confidence interval (CI) for dichotomous data and as the mean difference (MD) with the $95 \%$ CI for continuous data. The chi-square test and Higgins $\mathrm{I}^{2}$ test were used to assess heterogeneity. Additionally, we used Grading of Recommendations Assessment, Development, and Evaluation (GRADE) pro/GDT to assess the certainty of evidence (CoE) (21). Albatross plots were also generated using STATA/SE v.16.1 (StataCorp LLC, College Station, TX, USA) to visualize the effect of direction on the primary and secondary outcomes.

\section{Results}

\subsection{Descriptions of the Included Trials}

After the literature search, 2259 records were retrieved, and 1661 titles and abstracts were screened after removing duplicates. Fifty-three eligible articles were assessed, and 37 articles were eliminated for various reasons, leaving 16 full-text articles [23-38] that met 
the inclusion criteria for final inclusion (Figure 1). All included studies were conducted in China and published between 2002 and 2020 in the Chinese language.

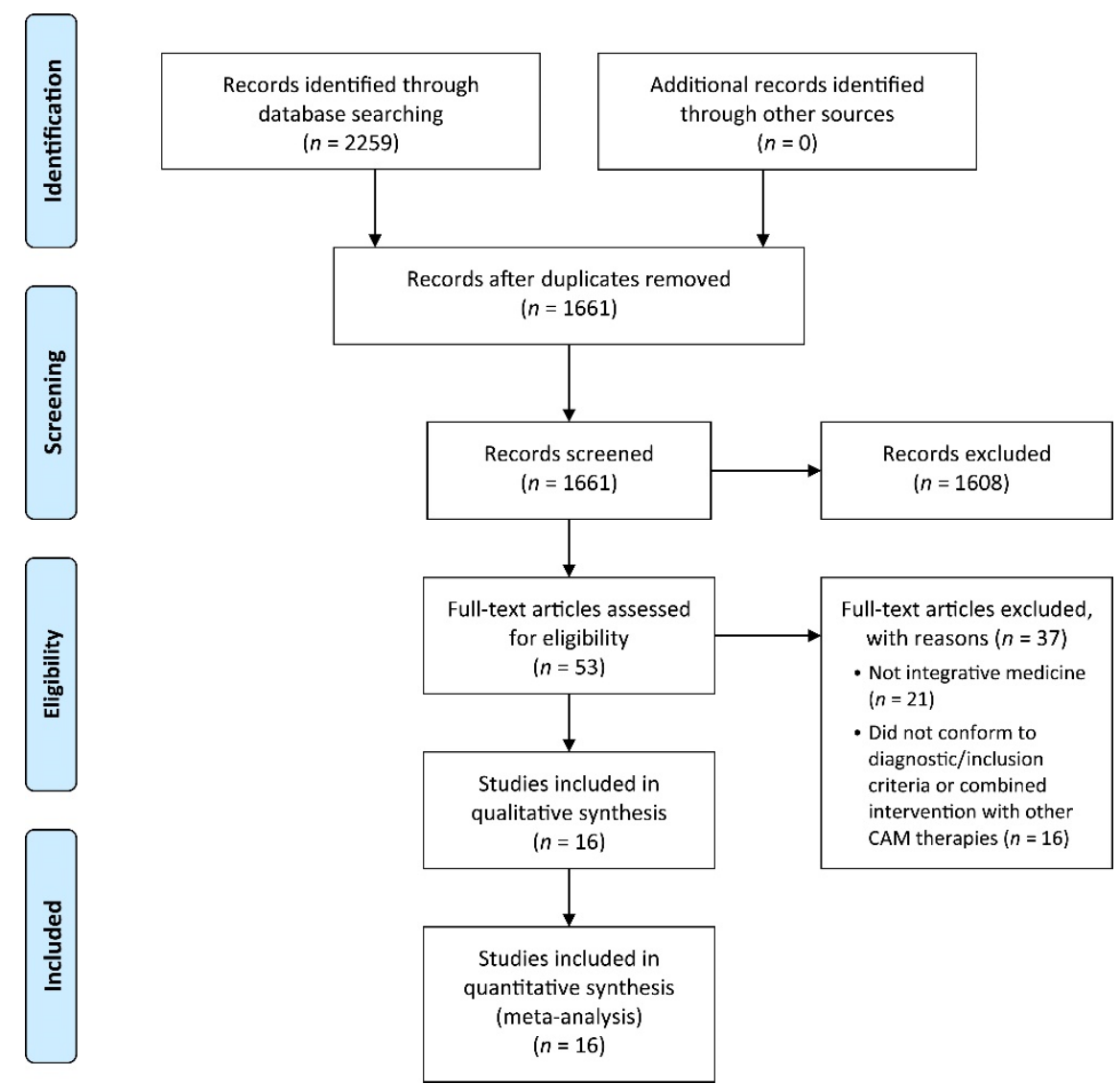

Figure 1. Flow chart of the study selection process.

All studies were two-arm parallel-designed trials. The mean age of patients from the included studies ranged from 23.4 to 41.3 years. The average disease duration ranged from 2.5 to 12.6 years. The total sample size was 987 , with the number of participants in each group ranging from 20 to 57 . All included studies used integrative medicine (combination of herbal medicine and drug therapy) as the intervention and drug therapy alone as the comparator. The details of the included studies are shown in Table 1.

Herbal medicine interventions in the included studies were herbal prescriptions given in the form of oral decoctions. Thirteen studies used standard prescriptions or modified prescriptions, whereas the remaining three studies used pattern identification (PI)-based prescriptions. The standard and modified prescriptions included modified Gancao Xiexin decoction, modified Qingdai san, Shen's Shengdi Qinlian Tufuling decoction, modified Huanglian decoction, modified Yiqi Jiedu Quyu prescription, standard/modified Huiyan Zhuyu decoction, Baitouweng decoction, Yinshenhao decoction, Duanxia Shenshi decoction, Longdan Xiegan decoction, modified Chixiaodou Danggui San, modified Ziyin Yuyang decoction, and Leiling Jiedu decoction. The herb with the highest frequency of use across the prescriptions was Glycyrrhizae Radix et Rhizoma, followed by Scutellariae Radix and Coptidis Rhizoma. The herbal compositions of the included prescriptions are presented in Table 2. The conventional drugs included thalidomide, celecoxib, azathioprine, prednisone, levamisole, and cyclophosphamide (all administered orally), and dexamethasone (administered intravenously). 
Table 1. Summary of randomized controlled trials on herbal medicine for the treatment of Behcet's disease.

\begin{tabular}{|c|c|c|c|c|c|c|}
\hline $\begin{array}{l}\text { First Author } \\
\text { (Year) [Ref] }\end{array}$ & $\begin{array}{c}\text { Sample Size (Ran- } \\
\text { domized/Analyzed) } \\
\text { Mean Age/Disease } \\
\text { Duration (Years) } \\
\text { Diagnostic Criteria }\end{array}$ & $\begin{array}{l}\text { Integrative } \\
\text { Medicine } \\
\text { (Regimen) }\end{array}$ & $\begin{array}{l}\text { Drug Therapy } \\
\text { (Regimen) }\end{array}$ & Main Outcomes & Results & Adverse Effect \\
\hline $\begin{array}{l}\text { Gong (2013) } \\
\text { [23] }\end{array}$ & $\begin{array}{l}\text { 60/60 } \\
\text { A: } 36.5 ; \text { B: } 36.3 / \text { n.r. } \\
\text { 1989 ISG; TCM } \\
\text { diagnosis }\end{array}$ & $\begin{array}{l}\text { (A) HM (Modified } \\
\text { Gancao Xiexin } \\
\text { decoction, } 2 \text { times } \\
\text { daily for } 3 \text { months, } \\
n=30 \text { ), plus (B) }\end{array}$ & $\begin{array}{l}\text { (B) } \\
\text { Thalidomide } \\
\text { (50 mg, once } \\
\text { daily for } 3 \\
\text { months, } n=30 \text { ) }\end{array}$ & $\begin{array}{l}\text { (1) Response rate } \\
\text { (2) Recurrence rate } \\
\text { (3) Symptom score } \\
\text { (4) ESR } \\
\text { (5) CRP }\end{array}$ & $\begin{array}{l}\text { (1) RR } 1.13 \text { [0.89, } 1.44], \mathrm{NS} \\
\text { (2) } 1 \text { month: RR } 0.33[0.04,3.03], \\
\text { NS; } 2 \text { months: RR } 0.25[0.06,1.08] \text {, } \\
\text { NS; } 3 \text { months: RR } 0.27[0.08,0.88] \text {, } \\
p=0.03 \\
\text { (3) Oral ulcer: MD } 0.14 \text { [-0.51, } \\
0.79], \text { NS; genital ulcer: MD } 0.11 \\
\text { [-0.56, 0.78], NS; eye } \\
\text { inflammation: MD }-0.06[-0.51 \text {, } \\
0.39], \text { NS; skin lesions: MD }-0.44 \\
\text { [-0.83, -0.05], } p=0.03 \\
\text { (4) MD } 0.10[-3.91,4.11], \mathrm{NS} \\
\text { (5) MD }-2.80[-10.29,4.69], \mathrm{NS}\end{array}$ & $\begin{array}{l}\text { Dizziness and } \\
\text { drowsiness (A: 1, } \\
\text { B: 5); dry mouth } \\
\text { and skin (A: } 1, \mathrm{~B} \text { : } \\
\text { 3); foreign body } \\
\text { sensation of skin } \\
\text { (B: } 1)\end{array}$ \\
\hline $\begin{array}{l}\text { Zhang } \\
\text { (2014a) [24] }\end{array}$ & $\begin{array}{l}\text { 60/60 } \\
\text { A: } 23.4 ; \text { B: } 24.8 / \mathrm{A}: \\
\text { 12.2; B: } 12.6 \\
\text { TCM diagnosis }\end{array}$ & $\begin{array}{l}\text { (A) HM (Modified } \\
\text { Qingdai san, } 2 \text { times } \\
\text { daily for } 2 \text { months, } \\
n=30 \text { ), plus (B) }\end{array}$ & $\begin{array}{l}\text { (B) } \\
\text { Thalidomide } \\
(50 \mathrm{mg} \text {, once } \\
\text { daily for } 2 \\
\text { months, } n=30)\end{array}$ & $\begin{array}{l}\text { (1) Response rate } \\
\text { (2) Recurrence rate } \\
\text { (3) ESR } \\
\text { (4) CRP }\end{array}$ & $\begin{array}{l}\text { (1) RR } 1.32[1.06,1.65], p=0.01 \\
\text { (2) } 2 \text { months: RR } 0.25[0.06,1.08] \text {, } \\
\text { NS } \\
\text { (3) MD } 0.23[-2.91,3.37], \text { NS } \\
\text { (4) MD }-0.80[-7.44,5.84], \text { NS }\end{array}$ & $\begin{array}{l}\text { Multiple neuritis } \\
\text { (B: 1) }\end{array}$ \\
\hline $\begin{array}{l}\text { Chen (2018) } \\
\text { [25] }\end{array}$ & $\begin{array}{l}\text { 68/68 } \\
\text { A: } 38.3 ; \text { B: } 39.5 / \text { A: } \\
\text { 3.8; B: } 3.9 \\
\text { CRA }\end{array}$ & $\begin{array}{l}\text { (A) HM (Shen's } \\
\text { Shengdi Qinlian } \\
\text { Tufuling decoction, } \\
2 \text { times daily for } 2 \\
\text { months, } n=34 \text { ), } \\
\text { plus (B) }\end{array}$ & $\begin{array}{l}\text { (B) } \\
\text { Thalidomide } \\
(25-50 \mathrm{mg} \text {, } \\
\text { once daily for } 2 \\
\text { months, } n=34)\end{array}$ & $\begin{array}{l}\text { (1) Response rate } \\
\text { (2) ESR } \\
\text { (3) CRP }\end{array}$ & $\begin{array}{l}\text { (1) RR } 1.10[0.94,1.30], \mathrm{NS} \\
\text { (2) MD }-6.10[-11.79,-0.41] \\
p=0.04 \\
\text { (3) MD }-1.80[-6.80,3.20], \mathrm{NS}\end{array}$ & $\begin{array}{l}\text { Constipation (A: } \\
\text { 1, B: 10); dizziness } \\
\text { (A: } 1, \mathrm{~B}: 4) ; \text { limb } \\
\text { numbness (B: 1); } \\
\text { nausea (B: 1) }\end{array}$ \\
\hline $\begin{array}{l}\text { Huang (2018) } \\
\text { [26] }\end{array}$ & $\begin{array}{l}\text { 60/60 } \\
\text { A: 38.0; B: } 41.3 / \mathrm{A}: \\
\text { 5.0; B: } 4.8 \\
\text { 2013 ICBD; } 2002 \\
\text { GCR-TCM }\end{array}$ & $\begin{array}{l}\text { (A) HM (Modified } \\
\text { Huanglian Wendan } \\
\text { decoction, } 2 \text { times } \\
\text { daily), plus (B) }\end{array}$ & $\begin{array}{l}\text { (B) } \\
\text { Thalidomide } \\
\text { ( } 50 \mathrm{mg}, 3 \text { times } \\
\text { daily; } \\
\text { Celecoxib } 0.2 \mathrm{~g} \text {, } \\
2 \text { times daily } \\
\text { for } 3 \text { months, } \\
n=30)\end{array}$ & $\begin{array}{l}\text { (1) Response rate } \\
\text { (2) Symptom score } \\
\text { (3) ESR } \\
\text { (4) CRP }\end{array}$ & $\begin{array}{l}\text { (1) RR } 1.17 \text { [0.93, } 1.48], \mathrm{NS} \\
\text { (2) Oral ulcer: MD }-0.63[-1.09 \text {, } \\
-0.17], p=0.007 \text {; genital ulcer: } \\
\text { MD }-0.73 \text { [-1.15, }-0.31] \\
p=0.0006 \text {; eye inflammation: MD } \\
-0.60[-1.11,-0.09], p=0.02 ; \\
\text { skin lesions: MD }-0.66[-1.15 \\
-0.17], p=0.009 \\
\text { (3) MD }-2.23[-4.07,-0.39] \\
p=0.02 \\
\text { (4) MD }-2.25[-4.16,-0.34] \\
p=0.02\end{array}$ & None \\
\hline $\begin{array}{l}\mathrm{Qu}(2016) \\
{[27]}\end{array}$ & $\begin{array}{l}\text { 40/40 } \\
\text { A: 37.0; B: } 37.3 / \mathrm{A}: \\
\text { 3.5; B: } 3.8 \\
\text { 1989 ISG; TCM } \\
\text { diagnosis }\end{array}$ & $\begin{array}{l}\text { (A) HM (Modified } \\
\text { Yiqi Jiedu Quyu } \\
\text { prescription, } 2 \text { times } \\
\text { daily for } 3 \text { months, } \\
n=20 \text { ), plus (B) }\end{array}$ & $\begin{array}{l}\text { (B) } \\
\text { Thalidomide } \\
\text { (50 mg, once } \\
\text { daily for } 3 \\
\text { months, } n=20 \text { ) }\end{array}$ & $\begin{array}{l}\text { (1) Response rate } \\
\text { (2) ESR } \\
\text { (3) CRP }\end{array}$ & $\begin{array}{l}\text { (1) RR } 1.12[0.91,1.38], \mathrm{NS} \\
\text { (2) MD }-7.32[-12.41,-2.23] \\
p=0.005 \\
\text { (3) } \mathrm{MD}-0.88[-5.89,4.13] \text {, NS }\end{array}$ & $\begin{array}{l}\text { Constipation (B: } \\
\text { 3); dizziness and } \\
\text { drowsiness (A: 1, } \\
\text { B: 2); } \\
\text { hypomenorrhea } \\
\text { (B: 1); pruritus (B: } \\
\text { 1) }\end{array}$ \\
\hline $\begin{array}{l}\text { Liu (2015) } \\
{[28]}\end{array}$ & $\begin{array}{l}46 / 43 \\
\text { A: } 35.8 ; \mathrm{B}: 35.7 / \mathrm{A}: \\
\text { 3.6; B: } 3.6 \\
\text { 1989 ISG }\end{array}$ & $\begin{array}{l}\text { (A) HM (Huiyan } \\
\text { Zhuyu decoction as } \\
\text { tea substitute for } 3 \\
\text { months, } n=22 \text { ), } \\
\text { plus (B) }\end{array}$ & $\begin{array}{l}\text { (B) } \\
\text { Thalidomide } \\
\text { ( } 75 \mathrm{mg} \text {, once } \\
\text { daily for } 3 \\
\text { months, } n=21 \text { ) }\end{array}$ & Response rate & $\operatorname{RR} 1.43[1.04,1.96], p=0.03$ & n.r. \\
\hline $\begin{array}{l}\mathrm{Ma}(2020) \\
{[29]}\end{array}$ & $\begin{array}{l}\text { 50/50 } \\
\text { A: } 37.3 ; \text { B: } 27.9 / \text { n.r. } \\
\text { 1989 ISG }\end{array}$ & $\begin{array}{l}\text { (A) HM (PI-based } \\
\text { prescription for } 3 \\
\text { months, } n=25) \\
\text { plus }(B)\end{array}$ & $\begin{array}{l}\text { (B) } \\
\text { Thalidomide } \\
(50 \sim 100 \mathrm{mg} \text {, } \\
\text { once daily for } 3 \\
\text { months, } n=25)\end{array}$ & $\begin{array}{l}\text { (1) Response rate } \\
\text { (2) ESR } \\
\text { (3) CRP }\end{array}$ & $\begin{array}{l}\text { (1) RR 1.21 [0.94, 1.55], NS } \\
\text { (2) Week 4: MD -1.00 [-4.35, } \\
\text { 2.35], NS; week 8: MD -10.00 } \\
\text { [-13.98, -6.02], } p<0.001 \text {; week } \\
\text { 12: MD -12.00 [-16.61, -7.39], } p \\
<0.001 \\
\text { (3) Week 4: MD - } 2.70[-3.79 \text {, } \\
\text {-1.61], } p<0.001 ; \text { week 8: } \\
\text { MD-1.40 [-2.54, }-0.26], p=0.02 \text {; } \\
\text { week 12: MD }-2.40[-3.47 \\
-1.33], p<0.001\end{array}$ & $\begin{array}{l}\text { Dizziness (A: 2; B: } \\
\text { 3); } \\
\text { leukocytopenia } \\
\text { (B: 1); liver } \\
\text { damage (A: 1; B: } \\
\text { 2); lower limb } \\
\text { numbness (B: 1) }\end{array}$ \\
\hline $\begin{array}{l}\text { Liu (2008) } \\
{[30]}\end{array}$ & $\begin{array}{l}\text { 100/100 } \\
\text { A: } 31 ; \text { B: 32/A: } 8.6 \text {; } \\
\text { B: } 8.4 \\
\text { 1987 ISG }\end{array}$ & $\begin{array}{l}\text { (A) HM (PI-based } \\
\text { prescription, } 2 \text { times } \\
\text { daily for } 3 \text { months, } \\
n=60) \text {, plus (B) }\end{array}$ & $\begin{array}{l}\text { (B) Prednisone } \\
(0.5 \sim 1 \mathrm{mg} / \mathrm{kg} \text {, } \\
\text { once daily for } 3 \\
\text { months, } n=40)\end{array}$ & Response rate & RR $1.21[1.06,1.38], p=0.006$ & n.r. \\
\hline Li (2011) [31] & $\begin{array}{l}\text { 60/60 } \\
\text { A: 30.0; B: 31.0/A: } \\
\text { 7.0; B: 8.0 } \\
\text { 1990 ISG }\end{array}$ & $\begin{array}{l}\text { (A) HM (PI-based } \\
\text { prescription, } 2 \text { times } \\
\text { daily for } 3 \text { months, } \\
n=30) \text {, plus (B) }\end{array}$ & $\begin{array}{l}\text { (B) Prednisone } \\
(0.5 \sim 1 \mathrm{mg} / \mathrm{kg} \text {, } \\
\text { once daily for } 3 \\
\text { months, } n=30)\end{array}$ & Response rate & $\operatorname{RR} 1.33[1.04,1.72], p=0.03$ & n.r. \\
\hline $\begin{array}{l}\text { Zhang } \\
\text { (2014b) [32] }\end{array}$ & $\begin{array}{l}80 / 80 \\
\text { A: 30.0; B: 32.0/A: } \\
\text { 7.0; B: 8.0 } \\
\text { 1990 ISG }\end{array}$ & $\begin{array}{l}\text { (A) HM } \\
\text { (Baitouweng } \\
\text { decoction }+ \\
\text { Yinchenhao } \\
\text { decoction, } 2 \text { times } \\
\text { daily for } 3 \text { months, } \\
n=40 \text { ), plus (B) }\end{array}$ & $\begin{array}{l}\text { (B) Prednisone } \\
(0.5 \sim 1 \mathrm{mg} / \mathrm{kg} \text {, } \\
\text { once daily for } 3 \\
\text { months, } n=40)\end{array}$ & Response rate & RR $1.04[0.82,1.32]$, NS & n.r. \\
\hline
\end{tabular}


Table 1. Cont.

\begin{tabular}{|c|c|c|c|c|c|c|}
\hline $\begin{array}{l}\text { First Author } \\
\text { (Year) [Ref] }\end{array}$ & $\begin{array}{c}\text { Sample Size (Ran- } \\
\text { domized/Analyzed) } \\
\text { Mean Age/Disease } \\
\text { Duration (Years) } \\
\text { Diagnostic Criteria }\end{array}$ & $\begin{array}{l}\text { Integrative } \\
\text { Medicine } \\
\text { (Regimen) }\end{array}$ & $\begin{array}{c}\text { Drug Therapy } \\
\text { (Regimen) }\end{array}$ & Main Outcomes & Results & Adverse Effect \\
\hline $\begin{array}{l}\text { Yan }(2019) \\
\text { [33] }\end{array}$ & $\begin{array}{l}30 / 30 \\
\text { A: 30.0; B: 32.0/A: } \\
\text { 7.9; B: 8.3 } \\
\text { 1990 ISG }\end{array}$ & $\begin{array}{l}\text { (A) HM (Duanxia } \\
\text { Shenshi decoction + } \\
\text { Longdan Xiegan } \\
\text { decoction, } 2 \text { times } \\
\text { daily for } 3 \text { months, } \\
n=30 \text { ), plus (B) }\end{array}$ & $\begin{array}{l}\text { (B) Prednisone } \\
\text { (0.5 1 mg/kg, } \\
\text { once daily for } 3 \\
\text { months, } n=30)\end{array}$ & Response rate & $\operatorname{RR} 1.33[1.04,1.72], p=0.03$ & n.r. \\
\hline Li (2016) [34] & $\begin{array}{l}\text { 114/114 } \\
\text { A: } 41.3 ; \text { B: } 41.3 / \text { n.r. } \\
\text { ISG }\end{array}$ & $\begin{array}{l}\text { (A) HM (Modified } \\
\text { Chixiaodou } \\
\text { Danggui San, } 2 \\
\text { times daily for } 2 \\
\text { weeks, } n=57 \text { ), plus } \\
\text { (B) }\end{array}$ & $\begin{array}{l}\text { (B) Prednisone } \\
\text { (20 60 mg, } \\
\text { once daily; } \\
\text { Levamisole } 50 \\
\text { mg, } 3 \text { times } \\
\text { daily for } 2 \\
\text { weeks, } n=57 \text { ) }\end{array}$ & Response rate & $\operatorname{RR} 1.20[1.04,1.39], p=0.02$ & n.r. \\
\hline $\begin{array}{l}\text { Zhu (2011) } \\
\text { [35] }\end{array}$ & $\begin{array}{l}\text { 67/67 } \\
\text { A: } 32.5 ; \text { B: } 30.0 / \text { n.r. } \\
1989 \text { ISG }\end{array}$ & $\begin{array}{l}\text { (A) HM (Modified } \\
\text { Ziyin Yuyang } \\
\text { decoction, } 2 \text { times } \\
\text { daily for } 2 \text { months, } \\
n=37 \text { ), plus (B) }\end{array}$ & $\begin{array}{l}\text { (B) Prednisone } \\
\text { (1 mg/kg once } \\
\text { daily) }+ \\
\text { Cyclophos- } \\
\text { phamide } 200 \\
\text { mg, } 3 \text { times } \\
\text { weekly for } 2 \\
\text { months, } n=30 \text { ) }\end{array}$ & Response rate & RR $1.20[0.96,1.49]$, NS & n.r. \\
\hline $\begin{array}{l}\text { Wang (2005) } \\
{[36]}\end{array}$ & $\begin{array}{l}42 / 42 \\
\text { A: } 34.2 ; \text { B: } 38.7 / \mathrm{A}: \\
\text { 3.6; B: } 3 \\
\text { 1989 ISG }\end{array}$ & $\begin{array}{l}\text { (A) HM (Leiling } \\
\text { Jiedu decoction, } 2 \\
\text { times daily for } 2 \\
\text { months, } n=22 \text { ), } \\
\text { plus (B) }\end{array}$ & $\begin{array}{l}\text { (B) Prednisone } \\
\text { (30 40 mg, } \\
\text { once daily for } 2 \\
\text { months) + Dex- } \\
\text { amethasone (10 } \\
\text { mg, once daily } \\
\text { for the 1st } \\
\text { week, } n=20)\end{array}$ & Response rate & RR $1.25[0.99,1.57]$, NS & None \\
\hline $\begin{array}{l}\text { Chang (2020) } \\
\text { [37] }\end{array}$ & $\begin{array}{l}\text { 60/60 } \\
\text { A: } 31.4 ; B: 32.07 / A: \\
\text { 2.48; B: } 2.51 \\
\text { 1989 ISG }\end{array}$ & $\begin{array}{l}\text { (A) HM (Modified } \\
\text { Huatan Quyu } \\
\text { prescription, } 2 \text { times } \\
\text { daily for } 2 \text { months, } \\
n=40 \text { ), plus (B) }\end{array}$ & $\begin{array}{l}\text { (B) } \\
\text { Azathioprine } \\
(100 \sim 150 \mathrm{mg}, \\
\text { once daily for } 2 \\
\text { months, } n=20)\end{array}$ & $\begin{array}{l}\text { (1) Response rate } \\
\text { (2) ESR } \\
\text { (3) CRP }\end{array}$ & $\begin{array}{l}\text { (1) RR } 1.12[0.92,1.36], \text { NS } \\
\text { (2) Reported only as } p<0.05 \\
\text { (3) Reported only as } p<0.05\end{array}$ & $\begin{array}{l}\text { Leukocytopenia } \\
\text { (A: 3; B: 4); liver } \\
\text { damage (A: 5; B: } \\
\text { 9); nausea and } \\
\text { vomiting (A: 2; B: } \\
\text { 3); skin rashes (A: } \\
\text { 2; B: 2) }\end{array}$ \\
\hline $\begin{array}{l}\mathrm{Gu}(2015) \\
{[38]}\end{array}$ & $\begin{array}{l}\text { 50/50 } \\
\text { A: } 30.2 ; \text { B: } 29.6 / \mathrm{A}: \\
2.5 ; \mathrm{B}: 2.3 \\
\text { CRA, TCM } \\
\text { diagnosis }\end{array}$ & $\begin{array}{l}\text { (A) HM (Modified } \\
\text { Huatan Quyu } \\
\text { prescription, } 2 \text { times } \\
\text { daily for } 2 \text { months, } \\
n=27 \text { ), plus (B) }\end{array}$ & $\begin{array}{l}\text { (B) } \\
\text { Azathioprine } \\
\text { (100 mg, once } \\
\text { daily for } 2 \\
\text { months, } n=23 \text { ) }\end{array}$ & $\begin{array}{l}\text { (1) Response rate } \\
\text { (2) CRP }\end{array}$ & $\begin{array}{l}\text { (1) RR } 1.11[0.93,1.32], \mathrm{NS} \\
\text { (2) MD }-2.87[-4.64,-1.10] \text {, } \\
p=0.002\end{array}$ & $\begin{array}{l}\text { Constipation (A: } \\
\text { 1); dizziness and } \\
\text { headache (B: } 2) ; \\
\text { drowsiness (A: 4); } \\
\text { edema (A: } 1) ; \\
\text { leukocytopenia } \\
\text { (A: 1, B: 1); loss of } \\
\text { appetite (B: 2); } \\
\text { nausea and } \\
\text { vomiting (B: 3); } \\
\text { peripheral } \\
\text { sensory } \\
\text { neuropathy (A: 2); } \\
\text { skin rashes (A: 1, } \\
\text { B: } 1 \text { ) }\end{array}$ \\
\hline
\end{tabular}

CRA: Chinese Rheumatology Association; CRP: C-reactive protein; ESR: erythrocyte sedimentation rate; GCR-TCM: Guiding principle of clinical research on new drugs of traditional Chinese medicine; HM: herbal medicine; ICBD: International Criteria for Behcet's disease; ISG: International Study Group Criteria; n.r.: not reported; NS: nonsignificant; PI: pattern identification; MD: mean difference; RR: risk ratio; TCM: traditional Chinese medicine. 
Table 2. Compositions of the herbal medicines for the treatment of Behcet's disease.

\begin{tabular}{|c|c|c|}
\hline Author (Year) [Ref] & Prescription & Composition of Herbs \\
\hline Gong (2013) [23] & $\begin{array}{l}\text { Modified Gancao Xiexin } \\
\text { decoction }\end{array}$ & $\begin{array}{l}\text { Glycyrrhizae Radix et Rhizoma } 9 \text { g, Glycyrrhizae Radix et Rhizoma } \\
\text { Praeparata } 9 \text { g, Scutellariae Radix } 9 \text { g, Coptidis Rhizoma } 6 \text { g, Angelicae } \\
\text { Gigantis Radix } 15 \text { g, Astragali Radix } 18 \text { g, Coicis Semen } 30 \text { g, Zingiberis } \\
\text { Rhizoma } 6 \text { g, Paeoniae Radix Rubra } 9 \text { g, Moutan Cortex } 9 \text { g, Lonicerae Flos } \\
20 \text { g, Forsythiae Fructus } 9 \text { g, Citri Pericarpium } 6 \text { g } \\
\text { Modification based on symptoms: [amenorrhea, forgetfulness, insomnia: } \\
\text { Ligustri lucidi Fructus } 20 \text { g, Polygoni Multiflori Ramulus } 20 \text { g]; [nausea, } \\
\text { vomiting, loss of appetite: Atractylodis Macrocephalae Rhizoma } 15 \text { g, } \\
\text { Amomi Fructus } 6 \text { g]; [vexing sensation in chest, palms and soles, tidal } \\
\text { fever: Anemarrhenae Rhizoma } 12 \text { g, Phellodendri Cortex } 6 \text { g]; [night } \\
\text { sweating or osteopyrexia: Lycii Radicis Cortex } 12 \text { g] }\end{array}$ \\
\hline Zhang (2014a) [24] & $\begin{array}{l}\text { Modified Qingdai san, } \\
\text { topical administration }\end{array}$ & $\begin{array}{l}\text { Indigo Naturalis, Coptidis Rhizoma, Phellodendri Cortex, Natrii Sulfas, } \\
\text { Bomeolum }\end{array}$ \\
\hline Chen (2018) [25] & $\begin{array}{l}\text { Shen's Shengdi Qinlian } \\
\text { Tufuling decoction }\end{array}$ & $\begin{array}{l}\text { Rehmanniae Radix } 30 \text { g, Scutellariae Radix } 30 \text { g, Coptidis Rhizoma } 6 \text { g, } \\
\text { Smilacis Glabrae Rhizoma } 30 \text { g, Caraganae Radix } 30 \text { g, Zedoariae Rhizoma } \\
30 \text { g, Paeoniae Radix Rubra } 15 \text { g, Moutan Cortex } 15 \text { g }\end{array}$ \\
\hline Huang (2018) [26] & $\begin{array}{l}\text { Modified Huanglian } \\
\text { Wendan decoction }\end{array}$ & $\begin{array}{l}\text { Pinelliae Rhizoma } 25 \text { g, Coptidis Rhizoma } 20 \text { g, Bupleuri Radix } 10 \text { g, Citri } \\
\text { Pericarpium } 15 \text { g, Atractylodis Macrocephalae Rhizoma } 20 \text { g, Lonicerae } \\
\text { Flos } 15 \text { g, Smilacis Glabrae Rhizoma } 20 \text { g, Plantaginis Semen } 10 \text { g, Moutan } \\
\text { Cortex } 15 \text { g, Glycyrrhizae Radix et Rhizoma } 10 \text { g }\end{array}$ \\
\hline Qu (2016) [27] & $\begin{array}{l}\text { Modified Yiqi Jiedu Quyu } \\
\text { prescription }\end{array}$ & $\begin{array}{l}\text { Astragali Radix } 30 \text { g, Rehmanniae Radix } 30 \text { g, Zedoariae Rhizoma } 15 \text { g, } \\
\text { Scutellariae Radix } 30 \text { g, Smilacis Glabrae Rhizoma } 30 \text { g, Caraganae Radix } \\
30 \text { g, Rhei Radix et Rhizoma } 9 \text { g, Glycyrrhizae Radix et Rhizoma } 12 \text { g, } \\
\text { Glycyrrhizae Radix et Rhizoma Praeparata } 12 \text { g } \\
\text { Modification based on symptoms: [erythema in the legs: Ranunculi Tuber } \\
15 \text { g, Forsythiae Fructus } 12 \text { g]; [vulvar ulcer: Millettiae Caulis } 30 \text { g, } \\
\text { Sophorae Radix } 15 \text { g]; [redness and pain in eyes: Bupleuri Radix } 9 \text { g, } \\
\text { Gardeniae Fructus } 9 \text { g]; [bitter taste in the mouth: Coptidis Rhizoma } 9 \text { g, } \\
\text { Lophatheri Herba } 9 \text { g]; [dry mouth, mild fever: Anemarrhenae Rhizoma } \\
9 \text { g, Phellodendri Cortex } 9 \text { g] }\end{array}$ \\
\hline
\end{tabular}

Rehmanniae Radix 50 g, Scrophulariae Radix 50 g, Aurantii Fructus 50 g, Persicae Semen 30 g, Angelicae Gigantis Radix 50 g, Carthami Flos 30 g, Bupleuri Radix 30 g, Paeoniae Radix Rubra 30 g, Platycodi Radix 30 g, Glycyrrhizae Radix et Rhizoma $30 \mathrm{~g}$

Huiyan Zhuyu decoction, as tea substitute
Modification based on PI: [dampness-heat pattern: Taraxaci Herba $20 \mathrm{~g}$, Moutan Cortex $30 \mathrm{~g}$ ]; [heat toxin pattern: Coicis Semen $50 \mathrm{~g}$, Scutellariae Radix 30 g]; [yin deficiency pattern: Anemarrhenae Rhizoma 50 g, Junci Medulla $10 \mathrm{~g}$, Ecliptae Herba $30 \mathrm{~g}$ ]; [spleen-stomach yin deficiency pattern: Astragali Radix 50 g, Codonopsis Pilosulae Radix 30 g]

(1) Lonicerae Flos 20 g, Chrysanthemi Flos 20 g, Violae Herba 20 g, Moutan Cortex 15 g, Gentianae Radix 15 g, Bupleuri Radix 15 g, Poria Sclerotium $20 \mathrm{~g}$, Coicis Semen $20 \mathrm{~g}$

(2) Glycyrrhizae Radix et Rhizoma $10 \mathrm{~g}$, Scutellariae Radix $15 \mathrm{~g}$, Zingiberis Rhizoma $10 \mathrm{~g}$, Coptidis Rhizoma $10 \mathrm{~g}$, Pinelliae Praeparatum cum Zingiberis $20 \mathrm{~g}$, Zizyphi Fructus 3 pieces

Xiaodu Yin (syndrome of retained dampness toxin) (2) Gancao Xiexin decoction (syndrome of Ma (2020) [29] retained dampness-heat) (3) Baihe Dihuang decoction/Modified Zhibai Dihuang decoction (syndrome of yin deficiency with inner heat)

Modification based on symptoms: [swelling and pain in joints: Millettiae
(3) Anemarrhenae Rhizoma 20 g, Phellodendron chinense Schneid. 15 g, Lilii Bulbus 20 g, Rehmanniae Radix 20 g, Poria Sclerotium 20 g, Chrysanthemi Flos $20 \mathrm{~g}$, Moutan Cortex $20 \mathrm{~g}$ Caulis 20 g, Gentiana macrophylla Pallas 20 g, Achyranthes japonica Nakai $20 \mathrm{~g}$, Sinomenium acutum $10 \mathrm{~g}$ ]; [erythema nodosum: Manis pentadactyla 15 g, Melandrium firmum Rohrbach 15 g, Paeoniae Radix Rubra 20 g]; [redness and blurriness in eyes: Celosia argentea $15 \mathrm{~g}$, Buddleja officinalis Maximowicz 15 g, Gardeniae Fructus 20 g]; [dry stools: Rhei Radix et Rhizoma 8 g, Magnoliae Cortex 15 g]; [insomnia: Polygonum multiflorum $20 \mathrm{~g}$, Ziziphus jujuba Mill.var.spinosa $20 \mathrm{~g}]$ 
Table 2. Cont.

\begin{tabular}{|c|c|c|}
\hline Author (Year) [Ref] & Prescription & Composition of Herbs \\
\hline Liu (2008) [30] & $\begin{array}{l}\text { (1) Xiegan san (syndrome } \\
\text { of retained dampness-heat } \\
\text { toxin) } \\
\text { (2) Danggui Liuhuang } \\
\text { decoction (syndrome of } \\
\text { yin deficiency with heat } \\
\text { toxin) } \\
\text { (3) Renshen Maidong san } \\
\text { (syndrome of dual } \\
\text { deficiency of qi and blood) }\end{array}$ & $\begin{array}{l}\text { (1) Scrophulariae Radix } 10 \text { g, Rhei Radix et Rhizoma } 10 \text { g, Scutellariae } \\
\text { Radix } 10 \text { g, Platycodi Radix } 10 \text { g, Angelicae Gigantis Radix } 10 \text { g, Natrii } \\
\text { Sulfas } 10 \text { g, Gentianae Radix } 10 \text { g, Plantaginis Semen } 15 \text { g, Notopterygii } \\
\text { Rhizoma } 6 \text { g, Anemarrhenae Rhizoma } 12 \text { g } \\
\text { (2) Angelicae Gigantis Radix } 10 \text { g, Rehmanniae Radix } 10 \text { g, Rehmanniae } \\
\text { Radix Praeparata } 10 \text { g, Coptidis Rhizoma } 10 \text { g, Scutellariae Radix } 10 \text { g, } \\
\text { Phellodendri Cortex } 10 \text { g, Astragali Radix } 20 \mathrm{~g} \\
\text { (3) Ginseng Radix } 10 \mathrm{~g} \text {, Atractylodis Rhizoma } 10 \text { g, Scutellariae Radix } 10 \text { g, } \\
\text { Anemarrhenae Rhizoma } 10 \text { g, Glycyrrhizae Radix et Rhizoma Praeparata } \\
10 \text { g, Bambusae Caulis in Taeniam } 10 \text { g, Rehmanniae Radix } 12 \text { g, Liriopis } \\
\text { Tuber } 20 \text { g }\end{array}$ \\
\hline Li (2011) [31] & $\begin{array}{l}\text { (1) Longgan Xiegan } \\
\text { decoction (syndrome of } \\
\text { retained dampness heat } \\
\text { toxin) } \\
\text { (2) Zhibai dihuang } \\
\text { decoction (syndrome of } \\
\text { yin deficiency with heat } \\
\text { toxin) } \\
\text { (3) Modified Shengmai yin } \\
\text { (syndrome of dual } \\
\text { deficiency of qi and blood) }\end{array}$ & $\begin{array}{l}\text { (1) Gentianae Radix } 12 \text { g, Scutellariae Radix } 12 \text { g, Rehmanniae Radix } 12 \text { g, } \\
\text { Bupleuri Radix } 10 \text { g, Bupleuri Radix } 10 \text { g, Alismatis Rhizoma } 10 \text { g, } \\
\text { Plantaginis Semen } 10 \text { g, Angelicae Gigantis Radix } 10 \text { g, Akebiae Caulis } 6 \text { g, } \\
\text { Glycyrrhizae Radix et Rhizoma Praeparata } 6 \text { g } \\
\text { (2) Anemarrhenae Rhizoma } 12 \text { g, Phellodendri Cortex } 12 \mathrm{~g} \text {, Rehmanniae } \\
\text { Radix Praeparata } 12 \text { g, Moutan Cortex } 12 \text { g, Dioscoreae Rhizoma } 12 \text { g, } \\
\text { Corni Fructus } 10 \text { g, Atractylodis Rhizoma } 10 \text { g, Alismatis Rhizoma } 10 \text { g } \\
\text { (3) Panacis Quinquefolii Radix } 6 \text { g, Schizandrae Fructus } 6 \text { g, Glycyrrhizae } \\
\text { Radix et Rhizoma Praeparata } 6 \text { g, Liriopis Tuber } 20 \text { g, Astragali Radix } 20 \text { g, } \\
\text { Ecliptae Herba } 20 \text { g }\end{array}$ \\
\hline Zhang (2014b) [32] & $\begin{array}{l}\text { Baitouweng decoction + } \\
\text { Yinchenhao decoction }\end{array}$ & $\begin{array}{l}\text { Pulsatillae Radix } 12 \text { g, Coptidis Rhizoma } 9 \text { g, Phellodendri Cortex } 9 \text { g, } \\
\text { Fraxini Cortex } 9 \text { g, Artemisiae Scopariae Herba } 30 \text { g, Rhei Radix et } \\
\text { Rhizoma9 g, Gardeniae Fructus } 9 \text { g }\end{array}$ \\
\hline Yan & $\begin{array}{l}\text { Duanxia Shenshi } \\
\text { decoction + Longdan } \\
\text { Xiegan decoction }\end{array}$ & $\begin{array}{l}\text { Atractylodis Rhizoma } 6 \text { g, Phellodendri Cortex } 9 \text { g, Polyporus } 9 \text { g, } \\
\text { Atractylodis Rhizoma } 9 \text { g, Crataegii Fructus } 9 \text { g, Lonicerae Flos } 12 \text { g, } \\
\text { Ailanthus Altissima Swingle } 12 \text { g, Sanguisorbae Radix } 9 \text { g, Gentianae } \\
\text { Radix } 9 \text { g, Angelicae Gigantis Radix } 9 \text { g, Gardeniae Fructus } 9 \text { g, Akebiae } \\
\text { Caulis } 6 \text { g, Plantaginis Semen } 6 \text { g, Plantaginis Semen } 6 \text { g, Scutellariae Radix } \\
9 \text { g, Glycyrrhizae Radix et Rhizoma } 6 \text { g, Rehmanniae Radix } 9 \text { g, Alismatis } \\
\text { Rhizoma } 9 \text { g }\end{array}$ \\
\hline Li (2016) [34] & $\begin{array}{l}\text { Modified Chixiaodou } \\
\text { Danggui San }\end{array}$ & $\begin{array}{l}\text { Phaseoli Semen } 15 \text { g, Angelicae Gigantis Radix } 15 \text { g, Scutellariae Radix } 6 \text { g, } \\
\text { Coptidis Rhizoma } 6 \text { g, Sophorae Radix } 6 \text { g, Plantaginis Semen } 6 \text { g, Akebiae } \\
\text { Caulis } 6 \text { g, Phyllostachys Folium } 6 \text { g, Glycyrrhizae Radix et Rhizoma } 6 \text { g }\end{array}$ \\
\hline Zhu (2011) [35] & $\begin{array}{l}\text { Modified Ziyin Yuyang } \\
\text { decoction }\end{array}$ & $\begin{array}{l}\text { Rehmanniae Radix Praeparata } 15 \text { g, Angelicae Gigantis Radix } 15 \text { g, } \\
\text { Anemarrhenae Rhizoma } 10 \text { g, Liriopis Tuber } 10 \text { g, Phellodendri Cortex } \\
10 \text { g, Cuscutae Semen } 9 \text { g, Ligustri Lucidi Fructus } 9 \text { g, Paeoniae Radix Alba } \\
9 \text { g, Moutan Cortex } 9 \text { g, Cinnamomi Cortex } 6 \text { g, Glycyrrhizae Radix et } \\
\text { Rhizoma } 6 \text { g } \\
\text { Modification based on PI: [dampness-heat pattern: Atractylodis Rhizoma } \\
10 \text { g; qi deficiency pattern: Astragali Radix } 15 \text { g] }\end{array}$ \\
\hline Wang (2005) [36] & Leiling Jiedu decoction & $\begin{array}{l}\text { Smilacis Glabrae Rhizoma } 15 \text { g, Codonopsis Pilosulae Radix } 15 \text { g, } \\
\text { Tripterygii Cortex } 10 \text { g, Angelicae Gigantis Radix } 15 \text { g, Salviae } \\
\text { Miltiorrhizae Radix } 10 \text { g, Lithospermi Radix } 15 \text { g, Rehmanniae Radix } 15 \text { g, } \\
\text { Oldenlandiae Diffusae Herba } 15 \text { g, Glycyrrhizae Radix et Rhizoma } 10 \text { g } \\
\text { Modification based on PI: [liver-kidney yin deficiency pattern: } \\
\text { Rehmanniae Radix Praeparata } 10 \text { g, Scrophulariae Radix } 10 \text { g, Lycii Fructus } \\
15 \text { g]; [spleen-kidney yang deficiency pattern: Aconiti Iateralis Radix } \\
\text { Praeparata } 10 \text { g, Cinnamomi Ramulus } 10 \text { g]; [dual deficiency of qi and } \\
\text { blood pattern: Astragali Radix } 10 \text { g, Atractylodis Macrocephalae Rhizoma } \\
10 \text { g] }\end{array}$ \\
\hline Chang (2020) [37] & $\begin{array}{l}\text { Modified Huatan Quyu } \\
\text { prescription }\end{array}$ & $\begin{array}{l}\text { Arisaema amurense Maximowicz var. serratum Nakai } 9 \text { g, Curcuma } \\
\text { phaeocaulis Valeton } 10 \text { g, Brassicae Semen } 10 \text { g, Pinelliae Rhizoma } 12 \text { g, } \\
\text { Angelicae Sinensis Radix } 12 \text { g, Persicae Semen 9g, Boswellia carterii } \\
\text { Birdwood } 10 \text { g, Commiphora myrrha } 9 \text { g, Zingiberis Rhizoma Recens } 15 \text { g, } \\
\text { Chuanxiong Rhizoma } 6 \text { g, Glycyrrhizae Radix et Rhizoma } 30 \text { g, Prunella } \\
\text { vulgaris Linné } 12 \text { g, Notopterygii Rhizoma seu Radix } 15 \text { g }\end{array}$ \\
\hline
\end{tabular}


Table 2. Cont.

\begin{tabular}{|c|c|c|}
\hline Author (Year) [Ref] & Prescription & Composition of Herbs \\
\hline Gu (2015) [38] & $\begin{array}{l}\text { Modified Huatan Quyu } \\
\text { prescription }\end{array}$ & $\begin{array}{l}\text { Pinelliae Rhizoma } 15 \mathrm{~g} \text {, Angelicae Gigantis Radix } 9 \text { g, Rehmanniae Radix } \\
9 \text { g, Atractylodis Rhizoma } 9 \text { g, Persicae Semen } 12 \text { g, Zingiberis Rhizoma } \\
\text { Recens } 25 \text { g, Paeoniae Radix Rubra } 9 \text { g, Cnidii Rhizoma } 6 \text { g, Citri } \\
\text { Pericarpium } 15 \text { g, Glycyrrhizae Radix et Rhizoma } 30 \text { g, Commelinae Herba } \\
12 \text { g, Carpesii Fructus } 15 \text { g, Euonymi Lignum Suberalatum } 12 \text { g, Zaocys } \\
\text { Praeparata } 12 \text { g } \\
\text { Modification based on PI: [liver dampness-heat pattern: Gentianae Radix, } \\
\text { Phellodendri Cortex, Phaseoli Semen] } \\
\text { Modification based on symptoms: [vulvar ulcer: Kochiae Fructus]; } \\
\text { [erosions of anus: Sophorae Fructus Praeparata]; [eye damage: Buddlejae } \\
\text { Flos, Cassiae Semen]; [mouth ulcer (external): Bomeolum, Borax, } \\
\text { Cinnabaris, Natrii Sulfas Exsiccatus] }\end{array}$ \\
\hline
\end{tabular}

\subsection{Risk of Bias}

The overall risk of bias was judged to be uncertain using Cochrane Collaboration's tool for risk of bias assessment (Figure 2).

Only four of the included studies reported the method used for random sequence generation, and none of the studies used allocation concealment methods [23-25,28]. As none of the studies reported the blinding of participants/personnel or outcome measurements, performance biases and detection biases for all studies were judged as unclear. Most studies were judged as having a low risk of bias because follow-up information or outcome data were missing; however, one study was judged as having a high risk of bias due to the incomplete report of several outcomes [37]. One study (27) that reported a few dropouts was eventually judged as having a low risk of bias, as the number of dropouts was less than $10 \%$ of the randomized samples, and the remaining studies did not report the percentage of patients who were lost to follow-up or did not perform an intention-to-treat (ITT) analysis [28]. The judgment of unclear risk of bias was given to all studies for reporting bias, as none of the studies had study protocols and did not provide sufficient information for further assessment. Information such as the source of funding, sample size calculation, and trial registration was also insufficient to assess other potential biases in the included studies. 
(A) Risk of bias graph

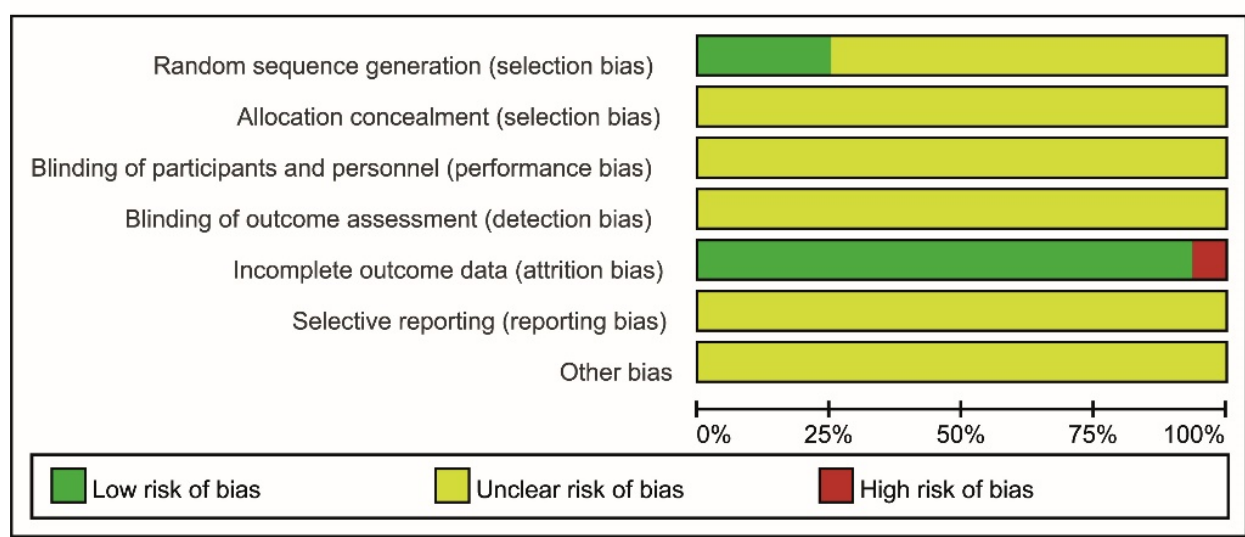

\section{(B) Risk of bias summary}

\begin{tabular}{|c|c|c|c|c|c|c|c|}
\hline & 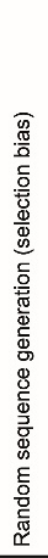 & 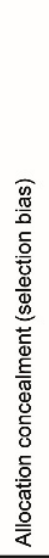 & 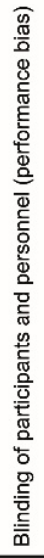 & 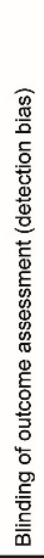 & 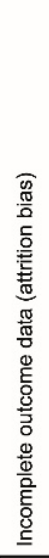 & 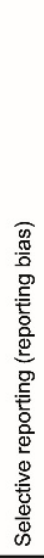 & 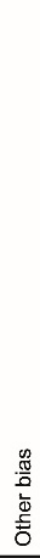 \\
\hline Chang (2020) & $?$ & $?$ & $?$ & $?$ & - & $?$ & $?$ \\
\hline Chen (2018) & + & $?$ & $?$ & $?$ & + & $?$ & $?$ \\
\hline Gong (2013) & + & $?$ & $?$ & $?$ & + & $?$ & $?$ \\
\hline $\mathrm{Gu}(2015)$ & $?$ & $?$ & $?$ & $?$ & + & $?$ & $?$ \\
\hline Huang (2018) & $?$ & $?$ & $?$ & $?$ & + & $?$ & $?$ \\
\hline $\mathrm{Li}(2011)$ & ? & $?$ & $?$ & $?$ & + & $?$ & $?$ \\
\hline $\mathrm{Li}(2016)$ & ? & $?$ & $?$ & $?$ & + & $?$ & $?$ \\
\hline Liu (2008) & $?$ & $?$ & $?$ & $?$ & + & $?$ & $?$ \\
\hline Liu (2015) & + & $?$ & $?$ & $?$ & + & $?$ & $?$ \\
\hline $\mathrm{Ma}(2020)$ & ? & $?$ & $?$ & $?$ & + & $?$ & $?$ \\
\hline Qu (2016) & ? & $?$ & $?$ & $?$ & + & $?$ & $?$ \\
\hline Wang (2005) & ? & $?$ & $?$ & $?$ & + & $?$ & $?$ \\
\hline Yan (2019) & $?$ & $?$ & $?$ & $?$ & + & $?$ & $?$ \\
\hline Zhang (2014a) & + & $?$ & $?$ & $?$ & + & $?$ & $?$ \\
\hline Zhang (2014b) & $?$ & $?$ & $?$ & $?$ & + & $?$ & $?$ \\
\hline Zhu (2011) & $?$ & $?$ & $?$ & $?$ & + & $?$ & $?$ \\
\hline
\end{tabular}

Figure 2. Risk of bias. (A) Risk of bias is shown as a graph: review authors' judgments on each item's risk of bias are presented as percentages across all included studies. (B) Risk of bias summary: review authors' judgments on each item's risk of bias for each included study. +: low risk of bias; -: high risk of bias; ?: unclear risk of bias. 


\subsection{Certainty of Evidence}

The CoE for each outcome as assessed using GRADE was low or very low. The 'Summary of findings' table on the main outcomes is presented in Table 3.

Table 3. Summary of Findings.

\begin{tabular}{|c|c|c|c|c|c|}
\hline \multicolumn{6}{|c|}{ Integrative Medicine Compared to Drug Therapy for Behcet's Disease } \\
\hline \multicolumn{6}{|c|}{$\begin{array}{l}\text { Patient or population: Patients with Behcet's disease } \\
\text { Setting: Hospital outpatients (Study design: randomized controlled trial) } \\
\text { Intervention: Integrative medicine } \\
\text { Comparison: Drug therapy }\end{array}$} \\
\hline \multirow[b]{2}{*}{ Outcome } & \multirow{2}{*}{$\begin{array}{l}\text { № of Participants } \\
\text { (Studies) } \\
\text { Follow-Up }\end{array}$} & \multirow[b]{2}{*}{$\begin{array}{l}\text { Certainty of } \\
\text { Evidence }\end{array}$} & \multirow[b]{2}{*}{$\begin{array}{l}\text { Relative Effect * } \\
(95 \% \text { CI })\end{array}$} & \multicolumn{2}{|c|}{ Anticipated Absolute Effects } \\
\hline & & & & $\begin{array}{c}\text { Risk with } \\
\text { Drug Therapy }\end{array}$ & $\begin{array}{l}\text { Risk Difference with } \\
\text { Integrative Medicine }\end{array}$ \\
\hline Response rate & $\begin{array}{l}1034 \\
(16 \mathrm{RCTs})\end{array}$ & $\begin{array}{l}\oplus \oplus \bigcirc \bigcirc \\
\mathrm{LOW}^{\mathrm{a}, \mathrm{b}}\end{array}$ & $\begin{array}{l}\text { RR } 1.19 \\
\text { (1.13 to } 1.25)\end{array}$ & 776 per 1000 & $\begin{array}{l}\mathbf{1 4 7} \text { more per } 1000 \\
\text { (101 more to } 194 \text { more) }\end{array}$ \\
\hline $\begin{array}{l}\text { Recurrence rate } \\
\text { ( } 2 \text { months) }\end{array}$ & $\begin{array}{l}120 \\
(2 \mathrm{RCTs})\end{array}$ & $\begin{array}{l}\oplus \oplus \bigcirc \bigcirc \\
\mathrm{LOW}^{\mathrm{a}, \mathrm{b}}\end{array}$ & $\begin{array}{l}\text { RR } 0.27 \\
(0.09 \text { to } 0.76)\end{array}$ & 250 per 1000 & $\begin{array}{l}\mathbf{1 8 3} \text { fewer per } 1000 \\
\text { ( } 228 \text { fewer to } 60 \text { fewer) }\end{array}$ \\
\hline Oral ulcers & $\begin{array}{l}120 \\
(2 \mathrm{RCTs})\end{array}$ & $\begin{array}{l}\oplus \bigcirc \bigcirc \bigcirc \\
\text { VERY LOW a,b,c }\end{array}$ & - & & $\begin{array}{l}\text { MD } 0.28 \text { lower } \\
\text { (1.03 lower to } 0.47 \\
\text { higher) }\end{array}$ \\
\hline Genital ulcers & $\begin{array}{l}120 \\
(2 \mathrm{RCTs})\end{array}$ & $\begin{array}{l}\oplus \bigcirc \bigcirc \bigcirc \\
\text { VERY LOW a,b,c }\end{array}$ & - & & $\begin{array}{l}\text { MD } 0.35 \text { lower } \\
\text { (1.17 lower to } 0.47 \\
\text { higher) }\end{array}$ \\
\hline Eye inflammation & $\begin{array}{l}120 \\
(2 \mathrm{RCTs})\end{array}$ & $\begin{array}{l}\oplus \bigcirc \bigcirc \bigcirc \\
\text { VERY LOW }\end{array}$ & - & & $\begin{array}{l}\text { MD } 0.32 \text { lower } \\
(0.84 \text { lower to } 0.21 \\
\text { higher })\end{array}$ \\
\hline Skin lesions & $\begin{array}{l}120 \\
(2 \mathrm{RCTs})\end{array}$ & $\begin{array}{l}\oplus \oplus \bigcirc \bigcirc \\
\mathrm{LOW}^{\mathrm{a}, \mathrm{b}}\end{array}$ & - & & $\begin{array}{l}\text { MD } 0.52 \text { lower } \\
(0.83 \text { lower to } 0.22 \\
\text { lower })\end{array}$ \\
\hline ESR & $\begin{array}{l}338 \\
(6 \mathrm{RCTs})\end{array}$ & $\begin{array}{l}\oplus \bigcirc \bigcirc \bigcirc \\
\text { VERY LOW } a, b, c\end{array}$ & - & & $\begin{array}{l}\text { MD 4.19 lower } \\
\text { (7.59 lower to } 0.79 \\
\text { lower) }\end{array}$ \\
\hline CRP & $\begin{array}{l}388 \\
\text { (7 RCTs) }\end{array}$ & $\begin{array}{l}\oplus \oplus \bigcirc \bigcirc \\
\mathrm{LOW}^{\mathrm{a}, \mathrm{b}}\end{array}$ & - & & $\begin{array}{l}\text { MD } 2.4 \text { lower } \\
\text { (3.19 lower to } 1.6 \text { lower) }\end{array}$ \\
\hline
\end{tabular}

* The risk in the intervention group (and its 95\% confidence interval) is based on the assumed risk in the comparison group and the relative effect of the intervention (and its 95\% CI). CI: confidence interval; MD: mean difference; RCT: randomized controlled trial; RR: risk ratio; a. Overall risk of bias is uncertain. Only four studies reported the simple randomization method, and the remaining studies did not provide relevant information. All studies reported a lack of allocation concealment and blinding. Other risk of bias domains were also concerning due to poor reporting. Therefore, the studies included were judged to have serious methodological limitations. ${ }^{\mathrm{b}}$. The sample size of each study is considered small, resulting in borderline imprecision. ${ }^{c}$. Heterogeneity across the studies is fairly high. GRADE Working Group grades of evidence. High certainty: We are very confident that the true effect lies close to that of the estimate of the effect. Moderate certainty: We are moderately confident in the effect estimate: the true effect is likely to be close to the estimate of the effect, but there is a possibility that it is substantially different. Low certainty: Our confidence in the effect estimate is limited: the true effect may be substantially different from the estimate of the effect. Very low certainty: We have very little confidence in the effect estimate: the true effect is likely to be substantially different from the estimate of effect.

\subsection{Outcome Measurements}

\subsubsection{Primary Outcomes}

Total Response Rate

Sixteen studies assessed the total response rate. Six studies reported a superior effect of integrative medicine compared with drug therapy [23-38], while the other ten studies reported equivalent effects between the two groups. The meta-analysis showed a favorable effect towards integrative medicine (RR 1.19, 95\% CI 1.13 to $1.25, n=1034, p<0.00001$, $\mathrm{I}^{2}=0 \%$, low CoE, Figure 3A). 


\section{(A) Response rate}

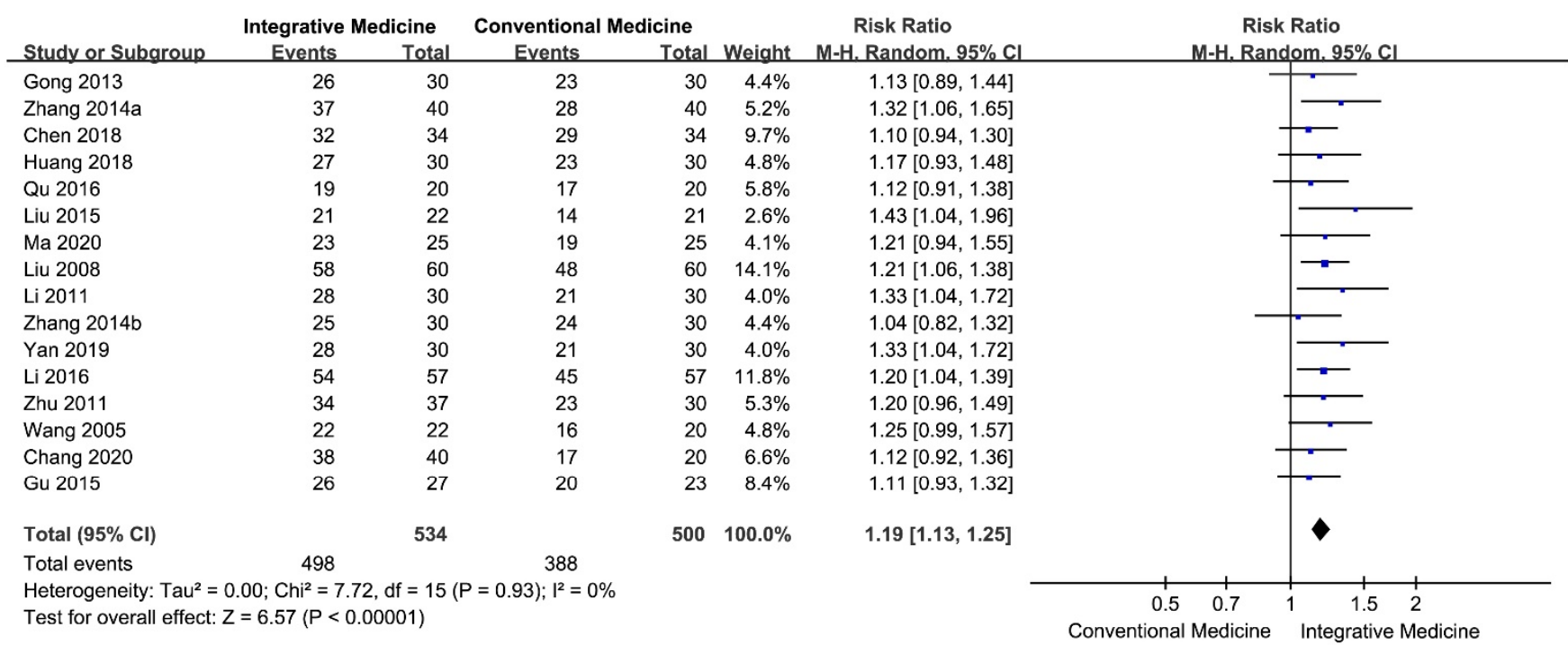

\section{(B) Recurrence rate}

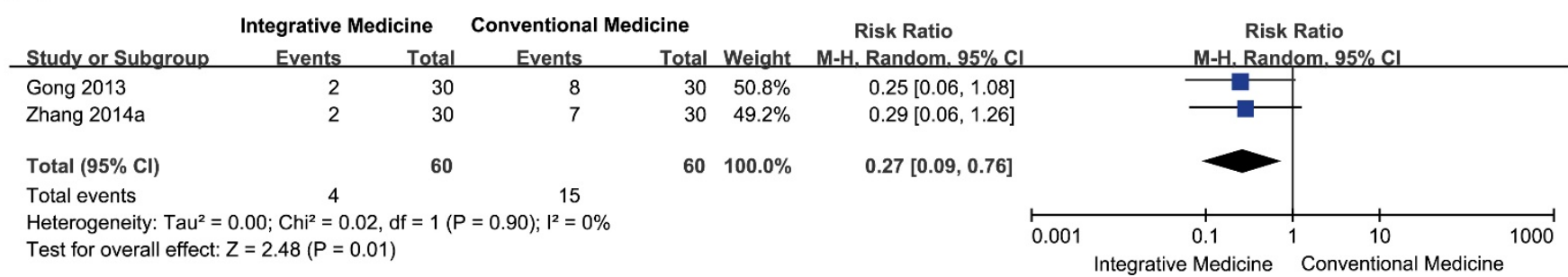

Figure 3. Forest plots of primary outcomes. (A) Total response rate; (B) recurrence rate.

Recurrence Rate

Only two studies that compared the effectiveness of herbal medicine combined with drug therapy with drug therapy assessed the recurrence rate [23,24]. One study [23] reported the recurrence rates between the two groups after 1 month, 2 months, and 3 months of follow-up. The recurrence rate was equivalent between the two groups after 1 month (RR 0.33, 95\% CI 0.04 to 3.03, $p=0.33$ ) and 2 months (RR $0.25,95 \%$ CI 0.06 to $1.08, p=0.06$ ) of follow-up. After 3 months of follow-up, the recurrence rate was lower for the herbal medicine combined with drug therapy group than for the drug therapy group (RR $0.27,95 \%$ CI 0.08 to $0.88, p=0.03$ ). Another study [24] reported the recurrence rate only at the 2-month follow-up (RR $0.25,95 \%$ CI 0.06 to $1.08, p=0.10$ ).

A meta-analysis of both studies on the recurrence rate after 2 months of followup showed that the group administered herbal medicine combined with drug therapy experienced a lower recurrence rate than the group administered drug therapy alone (RR $0.27,95 \%$ CI 0.09 to $0.76, n=120, p=0.01, \mathrm{I}^{2}=0 \%$, low CoE, Figure 3B).

\subsubsection{Secondary Outcomes}

Symptom Score

\section{Oral Ulcers}

Only two studies reported the symptom score for oral ulcers [23,26]. The meta-analysis showed an equivalent effect between the herbal medicine combined with drug therapy group and the drug therapy group (MD $-0.28,95 \% \mathrm{CI}-1.03$ to 0.47 , two studies, $n=120$, $p=0.47, \mathrm{I}^{2}=72 \%$, very low CoE, Figure $\left.4 \mathrm{~A}\right)$. 


\section{(A) Oral ulcer}

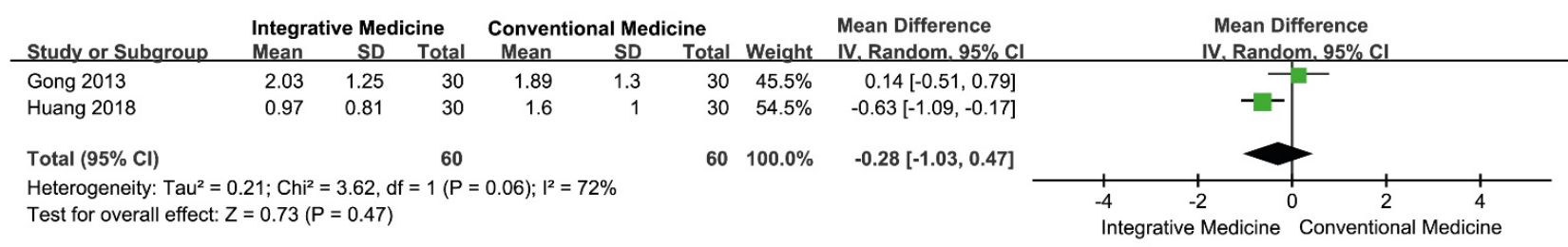

\section{(B) Genital ulcer}

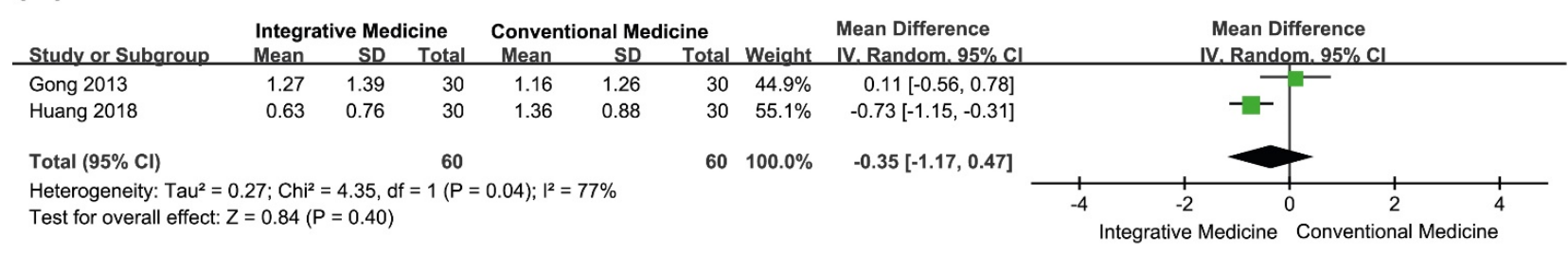

\section{(C) Eye inflammation}

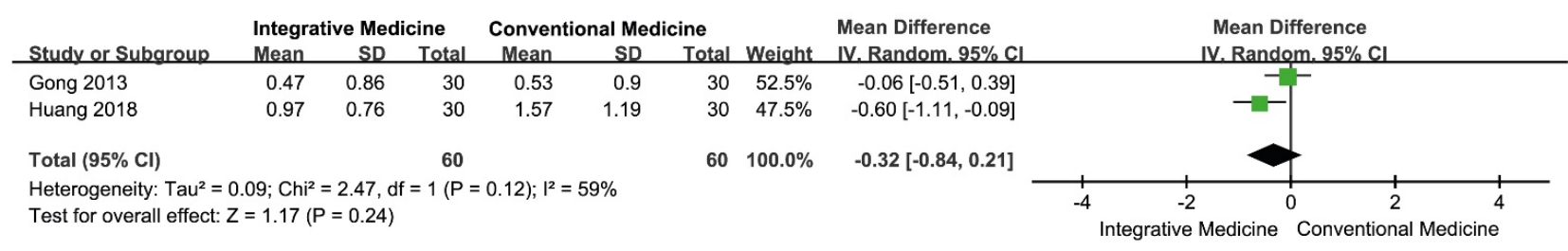

\section{(D) Skin lesion}

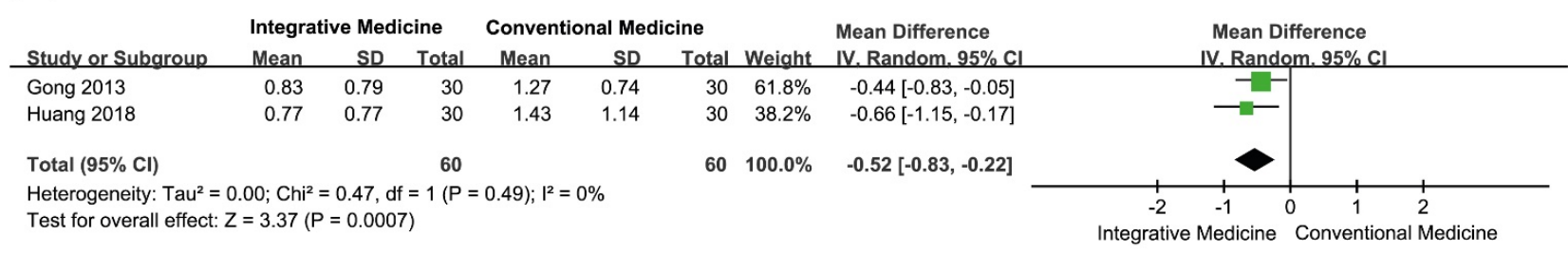

\section{(E) ESR}

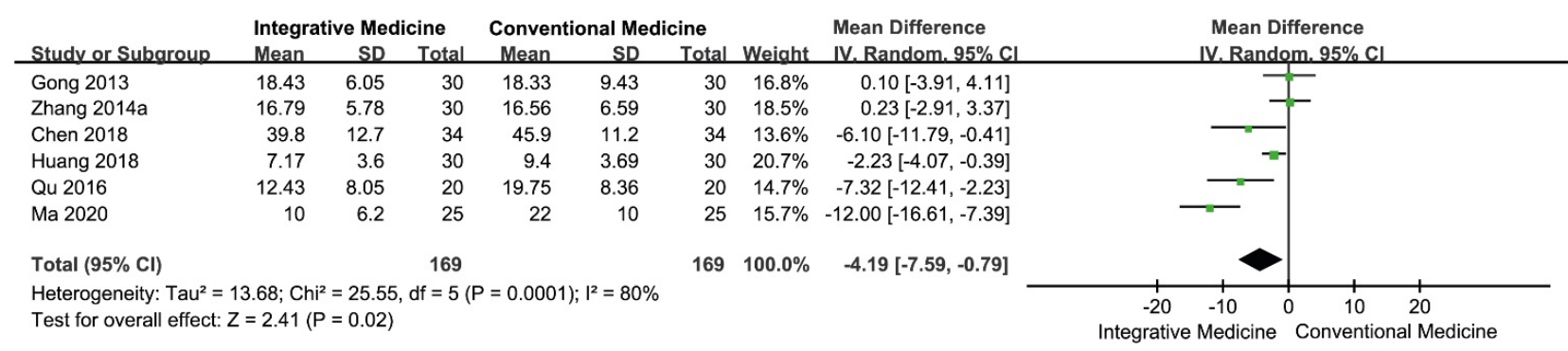

\section{(F) CRP}

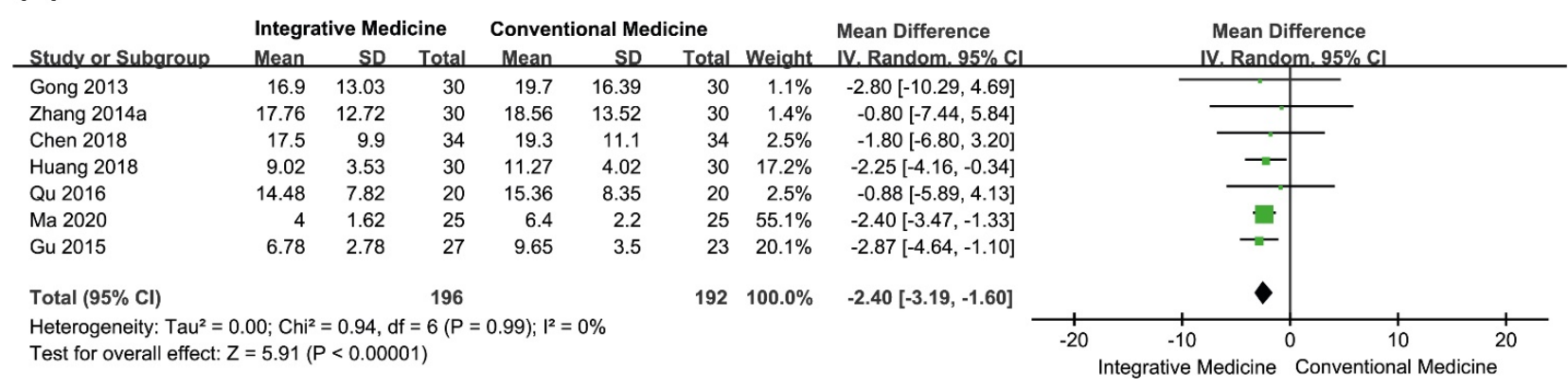

Figure 4. Forest plots of secondary outcomes. (A) Oral ulcers, (B) genital ulcers, (C) eye inflammation, (D) skin lesions, (E) ESR, (F) CRP. 


\section{Genital Ulcers}

Only two studies assessed the symptom score for genital ulcers $[23,26]$. The metaanalysis showed an equivalent effect between the herbal medicine combined with drug therapy group and the drug therapy group (MD $-0.35,95 \% \mathrm{CI}-1.17$ to $0.47, n=120$, $p=0.40, \mathrm{I}^{2}=77 \%$, very low CoE, Figure $\left.4 \mathrm{~B}\right)$.

Eye Inflammation

Only two studies evaluated the symptom score for eye inflammation [23,26]. The meta-analysis showed an equivalent effect between the herbal medicine combined with drug therapy group and the drug therapy group (MD $-0.32,95 \% \mathrm{CI}-0.84$ to $0.21, n=120$, $p=0.24, \mathrm{I}^{2}=59 \%$, very low CoE, Figure $4 \mathrm{C}$ ).

Erythrocyte Sedimentation Rate and C-Reactive Protein Levels

Four studies showed a favorable effect of herbal medicine combined with drug therapy compared to drug therapy alone [25-27,29], while two studies showed an equivalent effect between the two groups $[23,24]$. Of the six studies [23-27,29], one was excluded from the meta-analysis due to incomplete reporting [37]. The meta-analysis showed that the effect of herbal medicine combined with drug therapy on the ESR was superior to that of drug therapy alone (MD $-4.19,95 \% \mathrm{CI}-7.59$ to $-0.79, n=338, p=0.02, \mathrm{I}^{2}=80 \%$, very low CoE, Figure 4E).

Four studies showed a favorable effect of herbal medicine combined with drug therapy compared to drug therapy alone $[26,30,37,38]$, while four other studies showed an equivalent effect between the two groups [23-25,27]. Among the eight studies [23-27,30,37,38], one was excluded from the meta-analysis due to incomplete reporting [37]. The metaanalysis showed that herbal medicine combined with drug therapy had a superior effect on CRP levels (MD $-2.40,95 \%$ CI -3.19 to $-1.60, n=388, p<0.0001, \mathrm{I}^{2}=0 \%$, low CoE, Figure $4 \mathrm{~F}$ ) compared to drug therapy alone.

Adverse events

Nine studies assessed AEs [23-27,29,36-38]. Of the nine studies, two [26,36] reported no AEs for either integrative therapy or drug therapy. In the remaining seven studies, 30 AEs were reported in the herbal medicine combined with drug therapy group, and 67 AEs were reported in the drug therapy alone group. The details of the AEs are listed in Table 1.

\subsection{Albatross Plot}

The albatross plot showing the effects of direction and size range by $p$-value and the given sample sizes was generated for each included study (Figure 5, different outcome groups are presented in different colors). For the dichotomous data, the points were scattered across the contour lines (Figure 5A). All the points were clustered to the positive association side of the plot, implying that herbal medicine combined with drug therapy is favorable for the treatment of BD. For the continuous data, the points were scattered more towards the right side of the plot, with many points clustered around the null line, showing the equivalent effect of herbal medicine combined with drug therapy and drug therapy alone (Figure 5B). 
(A)

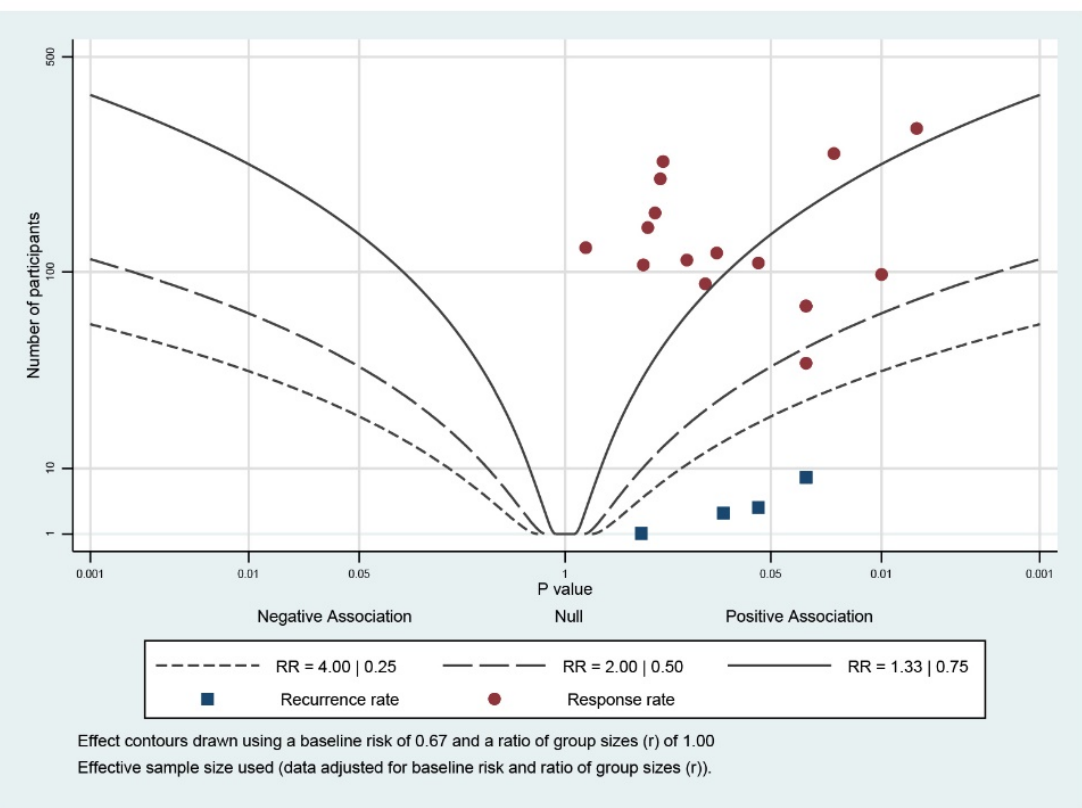

(B)

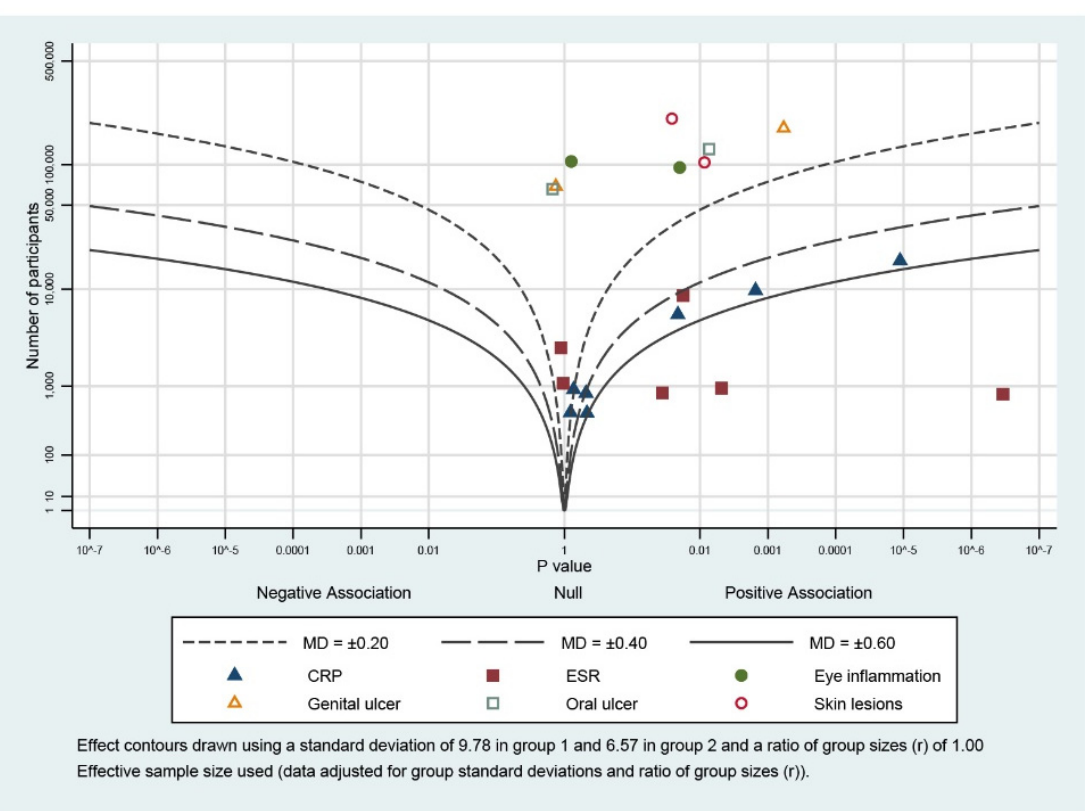

Figure 5. Albatross plot for (A) primary outcomes and (B) secondary outcomes.

\section{Discussion}

\subsection{Summary of the Main Results}

This systematic review revealed that herbal medicine combined with drug therapy appears to be more effective for the treatment of BD than drug therapy alone. Although the type of drug therapy used varied across the studies, herbal medicine combined with drug therapy was shown to improve the total response rate, skin lesions, ESR, and CRP level. However, the overall risk of bias of the studies was concerning, and the CoE was low. Information on AEs was also insufficient. In addition, the number of studies included for data synthesis for most outcomes was small. Future studies with rigorous RCTs may 
help establish the efficacy of herbal medicine combined with drug therapy in the treatment of BD.

\subsection{Quality of the Evidence}

The level of evidence as assessed by GRADE for the studies included in this metaanalysis was low. All the included studies had a high risk of bias. The evidence was downgraded by one level in the category of risk of bias due to a lack of information on the randomization, allocation concealment, and blinding methods in all studies. Further high heterogeneity of the symptom score outcome (oral ulcers, genital ulcers, and eye inflammation) downgraded the quality of evidence by one level in the category of inconsistency. In the category of indirectness, the included studies corresponded to the patient, intervention, comparison, outcomes (PICO) study, and it was ascertained that this effect was not serious. In the category of imprecision, the included studies had a small sample size, with 10 to 57 participants; thus, the quality of evidence was downgraded by one level. In general, the quality of reporting was poor in the included trials. Furthermore, the number of trials and total sample size included in our analysis were not sufficient to draw firm conclusions.

\subsection{Potential Biases in the Review Process}

There were several limitations to the present review. First, all the studies were published in China, limiting the overall generalization of the results. Despite searching eleven databases, all the studies that met the inclusion criteria were performed in China, where no studies with negative results have been reported [39]. Second, the methodological quality of the included studies was poor. There was a lack of information on randomization procedures and blinding methods that led to a concerning risk of bias. Third, the numbers of studies included in the meta-analyses of several outcomes, such as major BD symptoms and the recurrence rate, were small, resulting in a lack of significance and inconclusive results. In addition, the heterogeneity of herbal medicines used in combination with drug therapy highly limited the subgroup analysis and the comparison of therapeutic effects for herbal medicine combined with drug therapy to drug therapy alone. Such heterogeneity also makes it difficult to evaluate or identify herbal medicinal compounds effective for improving BD symptoms. Different types of integrative medicines and drug therapies used for different durations also made it difficult to compare the regimens. Fourth, herbal medicines used in the form of decoctions are not standardized and vary in their compositions and dosage, restricting the assessment of individual decoctions or single-herb effects. The diversity of herbal medicine administration within and across the included studies may have affected the overall results of our study, causing a certain degree of bias. Finally, we could not establish the safety of herbal medicine combined with drug therapy in treating $\mathrm{BD}$, as almost half of the included studies did not report AEs.

\subsection{Agreements and Disagreements with Other Studies or Reviews}

This review showed that herbal medicine combined with drug therapy is potentially effective for symptom relief in BD patients. Two previous reviews studied the effect of herbal medicine combined with drug therapy in the treatment of BD $[16,17]$. In our review, we found nine additional RCTs that adhered to standard clinical diagnostic criteria. The results of the analysis showed that the symptom improvement rate was higher with herbal medicine combined with drug therapy than with drug therapy alone. This result is similar to that of previous reviews, showing that the inclusion of additional studies did not change the significance of the results. Despite the addition of updated evidence, the small sample size and poor quality of the included studies remain the major limitations of our review.

\subsection{Implications for Clinical Practice}

Clinical manifestations vary greatly among BD patients, and conventional therapy highly depends on the severity of the disease, which often involves several body systems and organs. The main principle of BD treatment via drug therapy remains to be subduing 
inflammation during attacks and improving patients' quality of life by easing symptoms, increasing immunity, and reducing recurrence [40]. Although evidence supporting the use of azathioprine and cyclosporin A for ocular manifestations and interferon (IFN) $\alpha$ for mucocutaneous manifestations is available, evidence for vascular, gastrointestinal, and neurological involvement is still lacking [8]. To date, the effectiveness of drug therapy is based mostly on case reports/series and a limited number of RCTs.

Herbal medicine has been shown to have anti-inflammatory properties with favorable impacts on immune function and could play a critical role in complementing drug therapy [41]. The use of herbal medicine with anti-inflammatory properties also showed promising results in managing autoimmune diseases with oral manifestations, such as oral lichen planus, aphthous stomatitis, and Sjögren's syndrome, revealing the increasingly important role of herbal medicine in managing various inflammatory diseases [42-44].

Although evidence for proving the effectiveness of herbal medicine combined with drug therapy in the treatment of $\mathrm{BD}$ remains weak, accumulating evidence still shows encouraging effects. Medical professionals may still cautiously recommend the use of herbal medicine combined with drug therapy to patients who present side effects and resistance to drug therapy over a long duration.

\subsection{Implications for Research}

There are several concerns regarding the use of herbal medicine combined with drug therapy for the treatment of BD. First, the herbal prescriptions and drug therapies used in the included studies varied across studies. The included studies might not have been sufficient to demonstrate the complete effects for treating BD, and a subgroup analysis was unable to be conducted due to the small number of studies included. Second, all studies had a short duration of treatment. BD is an autoimmune disease with a high rate of recurrence. It is necessary to prove the effectiveness of herbal medicine combined with drug therapy on the recurrence rate by extending the period of treatment. Third, a number of AEs were reported in the herbal medicine combined with drug therapy group, and this has raised concerns about possible herb-drug interactions, such as pharmacokinetic and pharmacodynamic (PK-PD) interactions. The integration of herbal medicines with conventional drugs may cause changes in the movement, absorption, biochemical, and physiological effects of the herbs. Herb-drug interactions due to the concurrent use of herbs with conventional drugs may also magnify the effects of drugs, plausibly leading to the effectiveness of herbal medicine combined with drug therapy in treating BD. Fourth, the herbs with the highest frequency of use across the studies were Glycyrrhizae Radix et Rhizoma, Scutellariae Radix, and Coptidis Rhizoma. [45-47] These herbs have shown great potential in the treatment of inflammation-related diseases due to their anti-inflammatory active compounds. Future studies on these herbs, as single herbs or decoctions, should be considered to validate their usage and effectiveness in treating BD. In general, welldesigned RCTs in compliance with the CONSORT guidelines [48], long-term treatment periods, large sample sizes, and data on PK-PD parameters are warranted to guide the implementation of integrative medicine in clinical practice in the near future.

\section{Conclusions}

This review showed a significant improvement in symptoms upon the administration of herbal medicine combined with drug therapy in patients with BD. However, the included studies were performed on small sample sizes, had short treatment periods, and lacked detailed descriptions of the methodologies. To establish the effectiveness of herbal medicine combined with drug therapy in the treatment of $\mathrm{BD}$, future RCTs designed in compliance with the CONSORT guidelines are required to ensure a larger sample size, longer treatment duration, and rigorous evidence-based treatment methodology.

Supplementary Materials: The following are available online at https:/ /www.mdpi.com/article/10 $.3390 /$ pharmaceutics13040476/s1. 
Author Contributions: Conceptualization, J.H.J. and M.S.L.; methodology, J.H.J., L.A., and M.S.L.; software, J.H.J. and M.S.L.; validation, T.Y.C. and M.S.L.; formal analysis, L.A. and H.W.L.; investigation, J.H.J. and L.A.; resources, M.S.L.; data curation, H.W.L. and M.S.L.; writing-original draft preparation, J.H.J., L.A., and M.S.L.; writing—review and editing, H.W.L. and T.Y.C.; visualization, H.W.L. and M.S.L.; supervision, M.S.L.; project administration, M.S.L.; funding acquisition, M.S.L. All authors have read and agreed to the published version of the manuscript.

Funding: This research was supported by a grant from the Integrative Medicine Research Project through Wonkwang University Jangheung Integrative Medical Hospital, funded by the Ministry of Health and Welfare, Republic of Korea (grant number: 1465025215) and the Korea Institute of Oriental Medicine (KIOM) (G17250, KSN2013210, and KSN2021210). The funders had no role in the study design, data collection and analysis, decision to publish, or preparation of the manuscript. We received funds for open access publication fees from our institute, KIOM.

Institutional Review Board Statement: Not applicable.

Informed Consent Statement: Not applicable.

Data Availability Statement: Data sharing not applicable.

Conflicts of Interest: The authors have no conflict of interest to disclose.

\section{References}

1. Choi, H.; Seo, M.; Ryu, H.; Baek, H. Cross-cultural adaptation and validation of the Behcet's disease current activity form in Korea. Korean J. Intern. Med. 2015, 30, 714-718. [CrossRef]

2. Keino, H.; Okada, A. Behcet's disease: Global epidemiology of an old silk road disease. Br. J. Ophthalmol. 2007, 91, 1573-1574. [CrossRef]

3. Alpsoy, E.; Zouboulis, C.; Ehrlich, G. Mucocutaneous lesions of Behcet's disease. Yonsei Med. J. 2007, 48, 573-585. [CrossRef] [PubMed]

4. Calamia, K.; Wilson, F.; Icen, M.; Crowson, C.; Gabriel, S.; Kremers, H. Epidemiology and clinical characteristics of Behcet's disease in the US: A population-based study. Arthritis Rheum. 2009, 61, 600-604. [CrossRef] [PubMed]

5. Maldini, C.; Druce, K.; Basu, N.; LaValley, M.; Mahr, A. Exploring the variability in Behcet's disease prevalence: A meta-analytical approach. Rheumatology 2018, 57, 185-195. [CrossRef] [PubMed]

6. Saadoun, D.; Wechsler, B. Behcet's disease. Orphanet J. Rare Dis. 2012, 7, 20. [CrossRef]

7. Nava, F.; Ghilotti, F.; Maggi, L.; Hatemi, G.; Del Bianco, A.; Merlo, C.; Filippini, G.; Tramacere, I. Biologics, colchicine, corticosteroids, immunosuppressants and interferon-alpha for neuro-Behcet's syndrome. Cochrane Database Syst. Rev. 2014. [CrossRef]

8. Saleh, Z.; Arayssi, T. Update on the therapy of behçet disease. Ther. Adv. Chronic. Dis. 2014, 5, 112-134. [CrossRef]

9. Yurdakul, S.; Mat, C.; Tuzun, Y.; Ozyazgan, Y.; Hamuryudan, V.; Uysal, O.; Senocak, M.; Yazici, H. A double-blind trial of colchicine in Behcet's syndrome. Arthritis Rheum. 2001, 44, 2686-2692. [CrossRef]

10. National Center for Complementary and Integrative Health. Complementary, Alternative, or Integrative Health: What's in a Name? Available online: https:/ / nccih.nih.gov/health/integrative-health (accessed on 22 February 2021).

11. Tabish, S. Complementary and alternative healthcare: Is it evidence-based? Int. J. Health Sci. 2008, 2, V-IX.

12. Wang, J.; Xiong, X. Current situation and perspectives of clinical study in integrative medicine in China. Evid. Based Complement. Altern. Med. 2012, 2012, 268542. [CrossRef]

13. Sohn, S.; Bang, D.; Lee, S.; Kim, Y.; Lee, E.; Ha, J.; Kim, J.; Choi, S.; Lee, S. Combined treatment with colchicine and herba Taraxaci (tarazacum mongolicum Hand.-mazz.) attenuates Behcet's disease-like symptoms in mice and influences the expressions of cytokines. Int. Immunopharmacol. 2003, 3, 713-721. [CrossRef]

14. Sohn, S.; Bang, D.; Lee, S.; Kwon, H.; Lee, E.; Kim, J.; Choi, S.; Lee, S. Combined treatment of colchicine and herbal medicines (Gamichunghyulbohyul-tang of Gamiyongdamsagan-tang) attenuate the Behcet's disease symptoms in mice. J. Korean Med. 2001, 22, 102-108.

15. Jun, J.H.; Choi, T.Y.; Lee, H.W.; Ang, L.; Lee, M.S. Herbal Medicine for Behcet's Disease: A Systematic Review and Meta-Analysis. Nutrients 2020, 13, 46. [CrossRef]

16. Zhang, Z.; He, D.; Du, H.; Tian, J. Traditional medicine and integrative medicine for Behcet's disease: A meta analysis and systematic review. Sandong Med. 2014, 54, 91-94.

17. Zhang, Z.; Xu, J.; He, D. Effectiveness of combined with Chinese medicine and western medicine in the treatment of Behcet's disease: A meta-analysis. J. Gansu. Univ. Chin. Med. 2015, 32, 50-55.

18. International Study Group for Behçet's Disease. Criteria for diagnosis of Behcet's disease. Lancet 1990, 335, 1078-1080. [CrossRef]

19. National Administration of Traditional Chinese Medicine. Criteria of Diagnosis and Therapeutic Effect of Diseases and Syndromes in Traditional Chinese Medicine; Nanjing University Publishing House: Nanjing, China, 1994.

20. Chinese Rheumatology Association. Chinese guideline for the diagnosis and treatment of Behcet's disease. Chin. J. Rheumatol. 2011, 15, 345-347. [CrossRef] 
21. Zheng, X. Guiding Principle of Clinical Research on New Drugs of Traditional Chinese Medicine (Trial); China Medical Science Press: Beijing, China, 2002.

22. Higgins, J.; Altman, D.; Sterne, J. Chapter 8: Assessing risk of bias in included studies. In Cochrane Handbook for Systematic Reviews of Interventions Version 510; Higgins, J., Green, S., Eds.; Wiley: New York, NY, USA, 2015. Available online: http: / / www.cochrane-handbook.org (accessed on 21 January 2015).

23. Gong, Y. Clincal Study of Gancaoxiexintang and Thalidomide in the Treatment of Syndrome of Dampness-Heat in Behcet's Disease; Shandong University: Jinan, China, 2013; p. 20.

24. Zhang, Z. Clinical research on treating Behcet's syndrome with Qingdai San. CJCM 2014, 6, 121-123.

25. Chen, W.; Su, X.; Shen, P. Clinical observation of Shen's Shengdi Qinlian Tufuling decoction combined with thalidomide in the treatment of Behcet's disease. SH J TCM 2018, 52, 51-54.

26. Huang, H. Treatment of Liver and Spleen Damp Heat Type Behcet's Syndrome by Huanglian Wendan Decoction; Heilongjiang University: Harbin, China, 2018; p. 25.

27. Qu, H.; Xi, S.; Cao, Z.; Zeng, Y. Clinical observation of "Yiqi Jiedu Quyu decoction" and thalidomide in treating Behcet's disease. SH J TCM 2016, 50, 48-50.

28. Liu, L.; Shao, H.; Gao, M. The Epiglottis Zhuyu decoction on behalf of the tea treatment of oral cavity ulcer curative effect observation of Behcet's disease. Chin. J. Chin. Ethnomed. Ethnopharm. 2015, 24, 68-70.

29. Ma, H.; Liang, X.; Ge, X. Clinical observation on thalidomide combined with traditional Chinese medicine in the treatment of Behcet's Disease. Chin. Med. Mod. Distance Educ. China 2020, 18, 126-129.

30. Liu, S.; Wang, F. Observation on the curative effect of 60 cases of Behcet's disease treated with the combination of traditional Chinese and western medicine. Zhejiang J. TCM 2008, 43, 160.

31. Li, D.; Zhang, J. Observation on 30 cases of Behcet's disease treated with the combination of traditional Chinese and western medicine. Zhejiang J. TCM 2011, 46, 311.

32. Zhang, B.; Liu, S.; Peng, X. Clinical observation on treatment of Behcet's disease with Baitouweng decoction and Yinchenhao decoction combined with hormone. Chin. J. Tradit. Med. Sci. Technol. 2014, 21, 412.

33. Yan, J. Observation on treatment effect of integrative medicine on Behcet's disease. J. Pract. TCM 2019, 35, 1496-1497.

34. Li, H.; Duan, Y.; Liu, S. Clinical effect of Chixiaodou Danggui powder on Behcet's disease syndrome. Chin. J. Clin. Ration. Drug Use 2016, 9, 58-59.

35. Zhu, H.; Du, J. 37 cases of Behcet's disease treated with the combination of traditional Chinese and western medicine. Henan TCM 2011, 31, 1418.

36. Wang, X.; Meng, H.; Zeng, Z. Observation of therapeutic effect of Leilin deintoxication decoction united with combination of TCM and western medicine on 22 cases of Behect disease. Guiding J. TCM 2005, 11, 27-28.

37. Chang, J.; Wang, Y.; Hu, C.; Fu, P.; Wang, W.; Gu, Z. Clinical study of Chinese medicine combined with azathioprine tablets in the treatment of Behcet's disease. Electr. J. Clin. Med. Lit. 2020, 7, 29-30.

38. Gu, Z.; Wang, Y.; Hao, J.; Cao, Y.; Zeng, X.; Yang, X. Clinical obervation of chinese herbs for dissiating phlegm and removing blood stasis combined with azathioprine tablets on Behect's disease. Hebei J. TCM 2015, 37, 494-496.

39. Vickers, A.; Goyal, N.; Harland, R.; Rees, R. Do certain countries produce only positive results? A systematic review of controlled trials. Control. Clin. Trials 1998, 19, 159-166. [CrossRef]

40. Hatemi, G.; Silman, A.; Bang, D.; Bodaghi, B.; Chamberlain, A.M.; Gul, A.; Houman, M.H.; Kotter, I.; Olivieri, I.; Salvarani, C.; et al Management of Behcet disease: A systematic literature review for the European League Against Rheumatism evidence-based recommendations for the management of Behcet disease. Ann. Rheum. Dis. 2009, 68, 1528-1534. [CrossRef]

41. Pan, M.-H.; Chiou, Y.-S.; Tsai, M.-L.; Ho, C.-T. Anti-inflammatory activity of traditional Chinese medicinal herbs. J. Tradit. Complement. Med. 2011, 1, 8-24. [CrossRef]

42. Ghahremanlo, A.; Boroumand, N.; Ghazvini, K.; Hashemy, S.I. Herbal medicine in oral lichen planus. Phytother Res. 2019, 33, 288-293. [CrossRef]

43. Ali, S.; Wahbi, W. The efficacy of aloe vera in management of oral lichen planus: A systematic review and meta-analysis. Oral Dis. 2017, 23, 913-918. [CrossRef] [PubMed]

44. Zheng, L.W.; Hua, H.; Cheung, L.K. Traditional Chinese medicine and oral diseases: Today and tomorrow. Oral Dis. 2011, 17, 7-12. [CrossRef]

45. Yang, X.L.; Liu, D.; Bian, K.; Zhang, D.D. Study on in vitro anti-inflammatory activity of total flavonoids from Glycyrrhizae Radix et Rhizoma and its ingredients. Zhongguo Zhong Yao Za Zhi 2013, 38, 99-104. (In Chinese)

46. Li, C.; Lin, G.; Zuo, Z. Pharmacological effects and pharmacokinetics properties of Radix Scutellariae and its bioactive flavones. Biopharm Drug Dispos. 2011, 32, 427-445. [CrossRef]

47. Li, C.L.; Tan, L.H.; Wang, Y.F.; Luo, C.D.; Chen, H.B.; Lu, Q.; Li, Y.C.; Yang, X.B.; Chen, J.N.; Liu, Y.H.; et al. Comparison of anti-inflammatory effects of berberine, and its natural oxidative and reduced derivatives from Rhizoma Coptidis in vitro and in vivo. Phytomedicine 2019, 52, 272-283. [CrossRef] [PubMed]

48. Schulz, K.F.; Altman, D.G.; Moher, D. CONSORT 2010 statement: Updated guidelines for reporting parallel group randomised trials. BMJ 2010, 340, c332. [CrossRef] [PubMed] 